\title{
Cosmos visualized: Development of a qualitative framework for analyzing representations in cosmology education
}

\author{
Saeed Salimpour@* \\ Deakin University, 221 Burwood Highway, Burwood, Victoria 3125 Australia; \\ International Astronomical Union, Office of Astronomy for Education, \\ MPIA-Campus Königstuhl 17, D-69117 Heidelberg, Germany; \\ Haus der Astronomie, MPIA-Campus, Königstuhl 17, D-69117 Heidelberg, Germany, \\ and Max Planck Institut für Astronomie, Königstuhl 17, D-69117 Heidelberg, Germany \\ Russell Tytler@ \\ Deakin University, 221 Burwood Highway, Burwood, Victoria 3125, Australia \\ Urban Eriksson \\ Lund University Physics Education Research (LUPER) group, \\ Lund University, Box 118, 22100 Lund, Sweden \\ Michael Fitzgerald@ \\ Deakin University, 221 Burwood Highway, Burwood, Victoria 3125, Australia \\ and Las Cumbres Observatory, Goleta, California 93117, USA
}

(Received 14 October 2020; accepted 19 April 2021; published 24 May 2021)

\begin{abstract}
Our aesthetic response to the Universe, and the complexity of concepts through which we understand it, are inherently bound together in how we meaningfully interpret its nature. Over millennia the abstracted and intangible concepts of science have been developed and communicated through a rich array of representations across a variety of modes. The interpretation of such representations is a complex multidimensional and multimodal endeavor. This is particularly an issue in education where novices can struggle to engage with unfamiliar canonical representations. Learning in a discipline can be characterized as a process of developing disciplinary discernment in apprehending and using these representational systems. Using representations concerning the geometry of the Universe, evolution of the Universe, and cosmological expansion as examples, this paper provides an in-depth overview of the various multimodal representations through which concepts in cosmology are understood and communicated. In so doing this work unpacks the salient features of these representations in order to develop an underlying framework which we call the anatomy of representations (AOR). This study, in reviewing and analyzing representations in cosmology, explores this landscape of cosmology representations. This will allow for the characterization of how semiotic resources are mobilized, changed, and connections are made between various representational modes and levels, and an exploration of the landscape of cosmology and cosmology education. The AOR framework is intended as a guide for educators, including textbook authors, to support the development and interpretation of representations.
\end{abstract}

DOI: 10.1103/PhysRevPhysEducRes.17.013104

\section{INTRODUCTION}

Understanding the Universe, together with the objects it encompasses and the mechanisms that operate within it, can push our conceptual reasoning tools to their limits. This

\footnotetext{
*ssalimpour@deakin.edu.au

Published by the American Physical Society under the terms of the Creative Commons Attribution 4.0 International license. Further distribution of this work must maintain attribution to the author(s) and the published article's title, journal citation, and DOI.
}

is primarily owing to the vast spatial and temporal scales involved, coupled with the constant necessity to move between two-dimensional (2D), three-dimensional (3D), and four-dimensional (4D) projections [1]. Most importantly, unlike other sciences that allow scientists to create experiments within the laboratory, or travel to locations to observe the mechanisms or phenomena, astronomers cannot investigate causal links through experimental interventions. Astronomers must build knowledge through investigations of processes that occur outside of the possibility of manipulation. Astronomy, cosmology, paleontology, and evolutionary biology, referred to by Gray [2] 
as historical sciences, are differentiated from experimental sciences like physics. These historical sciences require reasoning that is "explanatory" or "reconstructive," "compared to predictive reasoning from causes to effects as is found in the experimental sciences" [2] (p. 5). However, the authors propose that it is more accurate to describe astronomy, astrophysics, and cosmology as being situated on the interface between historical and experimental sciences, in the sense that scientists engaged in these fields can do more than simply "observe" phenomena. Rather, with developments in computer technology they can run "experiments" by creating simulated universes that provide insights into the evolutionary history of the Universe and the objects within it [3]. Although it is argued that other sciences conduct simulations and build models, the nature and scale of those simulations and models are vastly different in astronomy.

Over millennia, scientists have developed a range of representations from the simple cave drawings of tens of thousands of years ago [4] to the complex data-driven computer simulations created in supercomputers of the 21st century (e.g., Ref. [3]). These representations have served many purposes from concept development, clarification, and problem solving to piquing curiosity in the broader public domain. The aims of data visualization, as highlighted by Norris [5], can be taken to apply to all representations. Essentially, the role of representations is to

- Allow the reader to gain a deeper tangible understanding of the concept.

- Make explicit features that would otherwise be implicit.

- Allow the reader to extract both quantitative and qualitative information.

- Allow the concept to be communicated to novices and experts.

In this work we define "reader" to be the individual who through observation and interpretation extracts meaning from a representation. This meaning could be at times different than the meaning intended by the creator of the representation [6], a circumstance we aim to unpack in this paper.

Scientific diagrams and representations in school textbooks can lead to conceptions not reflective of current science understandings. Furthermore, they can bolster preconceived experiential notions which are contrary to the science [7-9]. This challenge is exacerbated in astronomy, and especially cosmology, where concepts can be counterintuitive, involving complex temporal and spatial scale relations.

Science education in and for the 21 st century needs to reflect contemporary practices [10] and contemporary representational tools [11]. These include digital representations involving visualization $[12,13]$ and data visualization tools [14]. Further to this, curricula around the world are beginning to incorporate topics related to cosmology [15]. This requires the development and validation of tools and resources to help teachers take advantage of the rich potential afforded by these topics. Recent work [16] has revealed the range of conceptions students have about cosmology concepts, and the types of reasoning they use. It is increasingly understood that learning science involves induction into the representational practices of the discipline $[17,18]$. In the case of cosmology, these representational practices can be particularly challenging because of the scales involved, the counterintuitive nature of space time relations, and the data driven nature of the field. To support teachers to effectively implement representational practices in the classroom, we need to first understand the landscape of canonical representations in cosmology and then develop a framework that allows teachers, students, and textbook authors to unpack the meaning embodied in these representations.

This study is the first to review the range of canonical representations present in cosmology, particularly from an educational lens. Doing so allows us to characterize the processes by which disciplinary representations are manipulated or repurposed to be suitable for education. The aim is to develop a framework to help teachers and textbook authors link and unpack the key pedagogical and disciplinary features of representations in order to scaffold students in theirexploration and interrogation. The paper begins by contextualizing this study within cosmology. Following this we describe the various theoretical frameworks that underpin this study. Next, the qualitative methodology used to guide the analysis of the data is explained. The results are then presented together with the framework for analyzing cosmological representations. The paper concludes with a discussion of the key findings and implications for cosmology education.

\section{COSMOLOGY}

The science of cosmology historically has deep roots in mythology, culture, and religion [4]. For millennia our ancestors have looked up at the immense expanse of a sea of black (the night sky) with its myriad points of light (stars) and the white river that carves the expanse (the band of the Milky Way). Presented with this beauty and majesty, they asked questions about the fundamental nature of the Universe. These questions are asked to this day through both empirical and nonempirical lenses. Cosmology as a science aims to understand the mechanisms that operate to determine the large-scale structure of the Universe, its evolutionary history, and its future $[19,20]$. Cosmology encompasses complex spatial and temporal relations that are often counterintuitive. For example, exploring the Universe also provides us with an interesting perspective when talking about the notion of distance. Given the finite speed of the light $\left(\sim 300000 \mathrm{~km} \mathrm{~s}^{-1}\right)$, coupled with the vast distances in the Universe, we are in essence observing the past. This raises issues when measuring distances because distance is a measure between two locations at the same time. Therefore, cosmologists use a proxy that encapsulates both distance and time. This is encompassed by the parameter called redshift, usually denoted by the lowercase 
letter $z$. The further away an object the higher its redshift. For example, observations and measurements show that the galaxy GN-z11 has a redshift of 10.957 [21,22]. This translates to the observed light having been emitted from the galaxy when the Universe was around 400 million years old ( $\sim 3 \%$ of its current age). The use of the letter $z$ is a representational tool that encapsulates the complexity of distances in cosmology. To be able to appreciate the spatial and temporal relations encompassed by $z$ requires a significant level of disciplinary knowledge.

Cosmology in the past few decades has evolved into a precision science, driven by observation and simulations, and a continuous dialogue between experiment and theory. Observations by various ground and space-based telescopes have provided empirical data that is used to test and validate cosmological models (which are parametrized mathematically). These models make predictions which are then verified by observations. The concordance model in cosmology explains some of the key observations of the large-scale Universe. This model is called the lambda cold dark matter (LambdaCDM, $\Lambda$ CDM) model $[20,23,24]$ or the standard model of cosmology. CDM refers not to the temperature of dark matter, but to the motion of the potential particles associated with it-cold signifying slow moving; lambda $(\lambda)$ is referred to as the cosmological constant or vacuum energy and is associated with the accelerated expansion of the Universe [20]. The reason for this accelerated expansion is thought to be dark energy. The nature of dark matter and dark energy are still a topic of research. The $\Lambda \mathrm{CDM}$ model does have its challenges, and alternative models to $\Lambda \mathrm{CDM}$ exist [25].

The fundamental mathematical parametrization for representing our current understanding of cosmology is based on Einstein's field equations [26], from which Aleksander Friedmann $[27,28]$ derived a vital equation in cosmologythe Friedmann equation:

$$
\left(\frac{\dot{a}}{a}\right)^{2}=\frac{8 \pi G}{3 c^{2}} \epsilon-\frac{k c^{2}}{R_{0}^{2} a^{2}}+\frac{\Lambda}{3} .
$$

Equation (1) at its core talks about how matter, energy, and geometry interact in the Universe.

The left-hand side of the Friedmann equation essentially describes the expansion rate and is based on the HubbleLemaitre law [29,30]. Here the letter $a$ is technically referred to as the cosmic scale factor $a(t)$. The scale factor describes how the Universe, which is considered to be homogeneous (same everywhere) and isotropic (the same in every direction) on the largest scales (clusters of galaxies) expands over time. This assumption is verified by redshift surveys that show the large-scale distribution of galaxies. These surveys go back to the late 1970s, with CfA redshift survey [31,32], and more recent ones [33-35]. The right-hand side of Eq. (1) is a combination of several parameters related to the speed of light, gravity, matter, radiation, spatial curvature, and energy in the form of lambda $\Lambda$. This equation is presented as another instance of how representations in the form of mathematical notations are vital tools for describing the interactions and counterintuitive relations between space, time, energy, and matter. A considerable degree of disciplinary knowledge is required to understand and parse the notation used.

One of the seminal achievements in cosmology is the detection of the glow left over from when the Universe was around 380000 years old. The data from making measurements of this glow have been used to create what astronomers regard as one of the most, if not the most important images in cosmology - the cosmic microwave background radiation (CMB) (Fig. 1) [23,36,37]. This all-sky image is based on making measurements of how the temperature of the "sky" (referring to observations into the distant Universe) varies in a range of frequencies in the microwave region and extracting the faint signal of the $\mathrm{CMB}$. The image is a 2D representation of a 3D universe, created using the Mollweide map projection, which maps the entire sky in an oval shape maintaining the relative sizes and areas. The CMB is significant as it provides support for the model of an expanding Universe and allows key measurements (e.g., composition, geometry, overall density, and age of the Universe) to be made. This image is another level of representation that encompasses a depth of information that can only be explored and appreciated with the appropriate level of disciplinary knowledge.

In summary, cosmology uses many representations that are highly specialized and hence difficult for students to unpack, without expert knowledge in the field. One of the major challenges with bringing contemporary concepts of cosmology into the classroom is that some of the equations, such as Eq. (1), are not easily accessible to a general student with a predominantly high school mathematics background although they can be explained descriptively via high school level mathematics. Further, the representation in Fig. 1 contains lots of information that is not easily

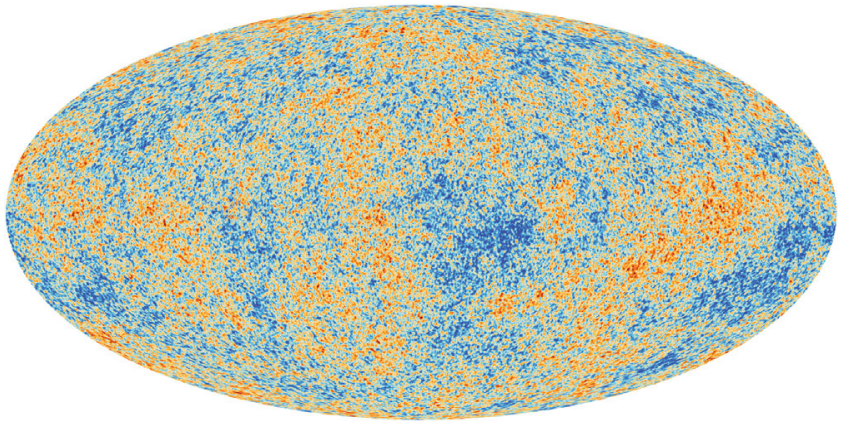

FIG. 1. Image of the cosmic microwave background. This is the faint glow left over from when the Universe was around 380000 years old. This representation was created based on data from the Planck satellite. Image credit: ESA/Planck Collaboration. 
discernible nor accessible to a student without a strong conceptual background in cosmology. To make these and other important scientific results and theories accessible to a high school student requires the appropriate use of representations targeted explicitly to provide this accessibility. This will be further explored in this paper.

\section{CONCEPTUAL FRAMEWORK}

This study draws on a range of theoretical perspectives. These include social semiotics [38-44], and within this disciplinary discernment $[1,45,46]$, representational competence [47,48], aesthetics [49], and structure mapping, which guides the use of analogies [50]. This collection of theoretical perspectives is brought together into a conceptual framework visualized in Fig. 2 and explained below.

The overarching lens underpinning this study is that of social semiotics, which concerns signs and meanings made in, and for a community or discipline. Extracting meaning from representations requires a level of disciplinary knowledge or, in relation to representations, discernment that is needed to guide how representations at a disciplinary level are repurposed to support school learning. Within the context of teaching and learning, social semiotics manifests in the construction and interrogation of representations. The teaching and learning of topics that have complex concepts, as is the case with cosmology, often requires the use of analogies and their associated representations. The effectiveness of analogies involves understanding the structural alignment between the elements and this can be analyzed using structure mapping. Finally, cosmological representations incorporate both science disciplinary and nondisciplinary aesthetics. This study in reviewing representations currently used in cosmology will use the following: social semiotics to understand the meaning of representations; disciplinary discernment to understand how representations can be interpreted at different levels; representational competence to situate these within teaching and learning; aesthetics to characterize values and judgments associated with the objects and processes of science, and in cosmology often the beauty and awe associated with astronomical objects and the vast scales being represented; and structure mapping to characterize the structural alignment of elements in analogical representations.

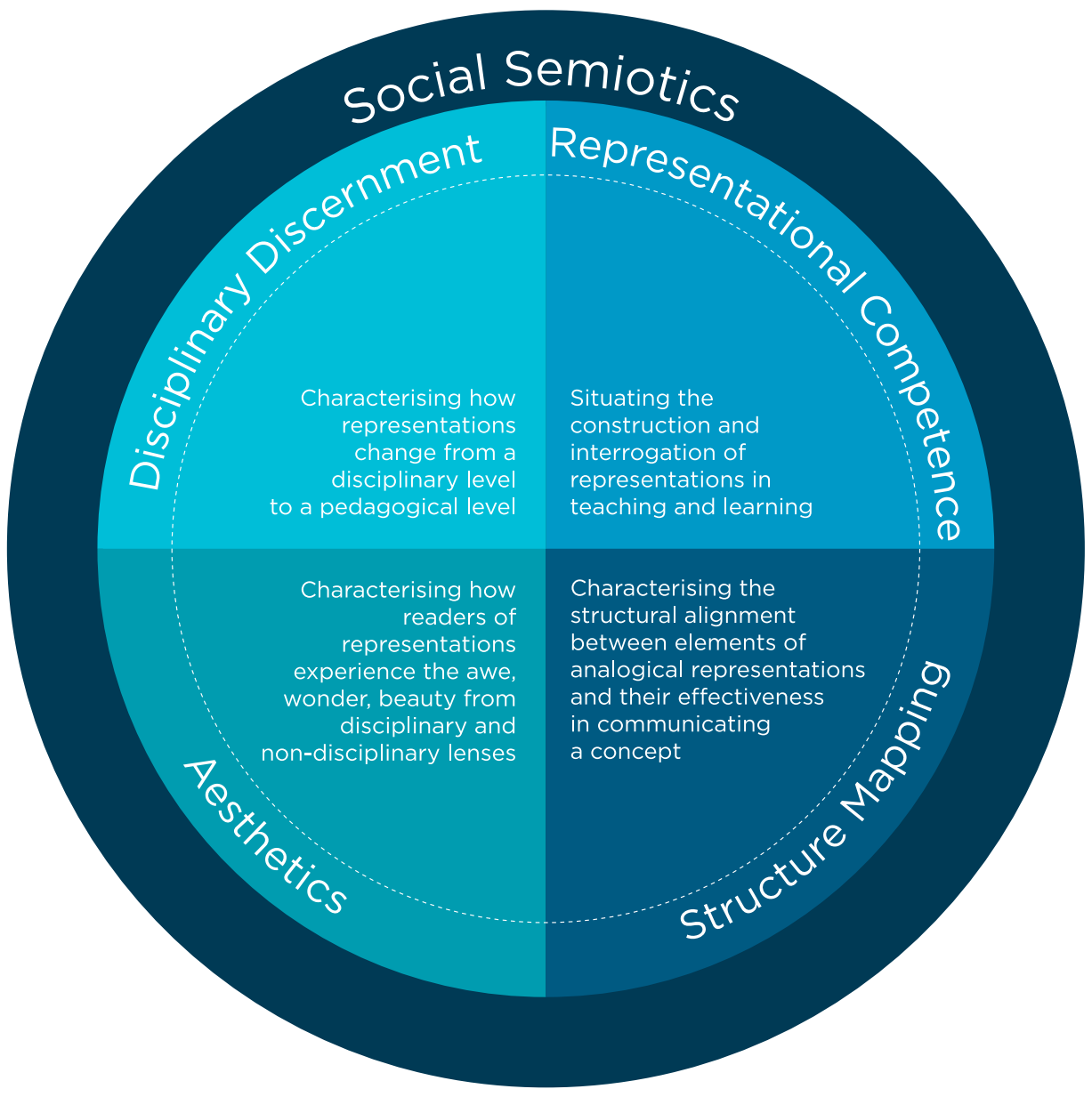

FIG. 2. The underlying conceptual framework of this study brings together four theoretical frameworks of disciplinary discernment, representational competence, aesthetics and structure mapping, within the overarching theoretical lens of social semiotics. 


\section{A. Social semiotics and representations}

This study focuses on the semiotic systems [38] through which cosmology is understood and communicated, including semiotic resources (representations, tools and activities) [51] in a range of forms. We begin by giving a brief overview of the theoretical underpinnings. Scientific concepts are constructed and understood through a range of representational practices involving symbolic, 2D mathematical and visual representations, and 3D models [52]. These representational or perceptual practices set the stage for this study. We recognize the growing literature in interpreting and using canonical representations $[12,48]$, and on students' construction of representations as a significant epistemic classroom practice [17,52-57]. Social semiotics explores semiosis, or the construction of meaning through signs, as related to the social norms of the community $[42,44]$. Therefore, meanings and meaning making go beyond the individual subjective realm to an activity involving the culture and communicative practices of the community. In the case of this study, the community is the astronomy discipline-specifically cosmology. This study is therefore framed as an investigation into the key features of the representation systems used for knowledge building and communication in cosmology, and the way semiotic systems (e.g., graphs, symbols, equations) are translated for and understood by the cosmology education community.

Distinguishing between the terms representations, models, and visualizations (RMVs) is a nontrivial endeavor since there are overlaps between the way these terms are used. Representations can be thought of as the encompassing term, with models and visualizations a subset of these [56]. Representations cover the range of drawings, sketches, equations, graphs, imagery, gestures that are used to make sense of and communicate ideas in science. RMVs at their core are reasoning tools with which explanations are generated and communicated and problems engaged with [55]. Representations are the material form through which abstract, often complex, concepts are made tangible and communicable, such as an annotated figure representing stellar evolution, or a graph representing temporal variation in a property. These representations often require a certain degree of disciplinary knowledge to be interpreted.

Models of a variety of types [57] permeate discovery and explanatory processes in science and science education and models can be categorized in various ways. For example, Harrison and Treagust [58] categorize models into four broad categories. The first three are of particular interest in this study:

- Scientific and teaching models

- Pedagogical analogical models that build conceptual knowledge

- Models depicting multiple concepts and/or processes

- Personal models of reality, theories and processes.

Gilbert [59] (p. 5) uses a different classification system for models, which describes six types:
- concrete models (for example, scale models, figurines);

- Pictorial or graphic models (for example, blueprints, photographs, diagrams);

- mathematical models (for example, formulas, graphs, topographic maps);

- verbal models (for example, descriptions, scripts, directions);

- simulation models (for example, simulation games, crash test dummies); and

- symbolic models (semiotic models) (for example, words, numbers, mathematics figures).

Visualization is increasingly recognized as central to scientific discovery processes [60] and to the teaching and learning of science [61]. Visualizations, or visual representations [62] form a subset of representations that focus on visuospatial features, i.e., representations that can have both visual and spatial features. Representations are crucial reasoning tools for learning and applying scientific concepts, and their interpretation, construction, and evaluation are key concerns underpinning this study. There are particular demands on teaching a contemporary science like cosmology due to the fact that (a) it is so new to curricula that there has not as yet been the time to establish pedagogical representations that will structure students' knowledge building, unlike topics like mechanics or optics; and (b) like many contemporary topics, cosmology has particular features (e.g., size, scale, spacetime relations) that are particularly challenging, and often counterintuitive.

Representational competence [48], sitting within a social semiotic theoretical framework, attends to the practicalities of student learning in classrooms [17]. It is premised on the notion that understanding and problem solving in cosmology as with other disciplines involves the interpretation, construction, evaluation, and coordination of representations. The interpretation of representations involves disciplinary discernment, including an appreciation of the structure and function of representations, and their partial but interconnecting nature. This provides a major focus for the analysis.

\section{B. Disciplinary discernment}

Representations act as tools in the teaching and learning process, with the learning process involving "noticing" and "reflection" on features of presented representations [45]. The student goes through the journey from noticing to reflection, eventually making sense of the new knowledge by assimilating it into their ways of conceptualizing phenomena, as part of their journey into developing the fluency in disciplinary discursive practices that counts as disciplinary knowledge. This process is encapsulated in the theoretical framework of disciplinary discernment [46].

Disciplinary discernment is closely linked to the concept of disciplinary affordances $[63,65]$, which is an important aspect in the reading of representations. This notion-reading-is 
expanded in the work of Eriksson [45,64]. In order to extract the intended meaning of the representation, which is specific to the discipline, students need to have a certain level of disciplinary discernment. Affordance refers to the inherent characteristics, or aspects, of a representation that constrains attention and opens up perspectives on a phenomenon. Disciplinary affordance is defined as "the agreed meaning making functions that a semiotic resource fulfils for a particular disciplinary community" [65] (p. 99). Therefore, affordance is related to a specific representation, while discernment is related to the student, or more appropriately, the reader. How the student attains the required level of disciplinary discernment is part of the learning process.

Furthermore, this notion of disciplinary affordance also has a companion-pedagogical affordance. This is defined as "The aptness of a semiotic resource for teaching some particular educational content" [65] (p. 18). In the context of this study, it can be defined as the need to provide ways of transforming disciplinary representations into representations that are geared towards structures that students notice as they are inducted into being able to read the disciplinary affordances of canonical semiotic resources. Similar to disciplinary affordance, pedagogical affordance is related to the representation itself.

\section{Aesthetics}

We argue in this section that aesthetics plays a particular and special role in astronomy or cosmology which has since the beginnings of time inspired awe, and speculation about our place in the Universe. This is reflected in astronomical representations, for instance, images of a galaxy, nebula or the night sky which can elicit some very deep aesthetic responses [66-70]. Feelings of wonder, or curiosity triggered by such representations are independent of whether the viewer understands the underlying science of how the representation was created or what is happening in the image; cf. disciplinary discernment [64]. As we will discuss in this section, there are three types of aesthetic response at play in representations of astronomy or cosmology phenomena; the everyday aesthetics we introduced above, the artrelated aesthetics expressed in terms of beauty, symmetry, color, shape, form, expressed through the language of visual arts $[69,70]$, and the scientific disciplinary aesthetics, which we define as aesthetics related to disciplinary practices and values such as clarity (e.g., of a graph, an equation), theoretical elegance and basis in evidence.

The notion of disciplinary aesthetics has its theoretical foundations in the work of the pragmatist John Dewey [71-73], who argued for a continuity between conceptual and aesthetic experience as an essential part of the learning process, linking conceptual learning fundamentally to aesthetics. Lemke [74] argues that you cannot have meaning without feeling. Wickman [49] argues that part of discernment in any scientific discipline is the aesthetics associated with the conceptual content and the representational systems underpinning this. Developing disciplinary discernment in relation to a representation involves coming to appreciate or value it and its purpose. This could apply to appreciating the clarity of a graph, or of the insights provided by a theory. In unpacking a representation, we need to be aware of the different aesthetics that are operating. The aesthetic response to a representation will depend on the viewer's level of disciplinary discernment, which will shape the nature and focus of the aesthetic experience. In the following sections we will unpack the way representations can trigger different types of aesthetic response.

\section{Aesthetic interactions}

Figures 3-7 provide examples of the various aesthetics and their interactions - the everyday aesthetic, the art aesthetic, and the disciplinary aesthetic. Although these images (Figs. 3, 4, 7) may be associated with astronomy, we should emphasize that the fields of astronomy, astrophysics, and cosmology are symbiotic. To understand cosmology, we need to understand the objects within the Universe. These images can, for instance, inspire us to ask cosmological questions about the Universe. Furthermore, our understanding of the scale of the Universe has changed with time, and so the objects and phenomena we consider cosmological have changed. Figure 3 shows an image of the night sky landscape, which is all too familiar to those living away from the glare of the city lights. This image is in ways tangible and accessible, involving an everyday aesthetic associated with feelings of beauty and grandeur. It also possesses an art aesthetic involving the arrangement of the photographic elements.

Figure 4 presents an image (representation) taken by the Hubble Space Telescope of the Sombrero Galaxy, located around 29 million light years away. This image alongside the everyday and art aesthetic encompasses a deeper disciplinary aesthetic in that the image reveals elements that reflect the history of our understandings of different

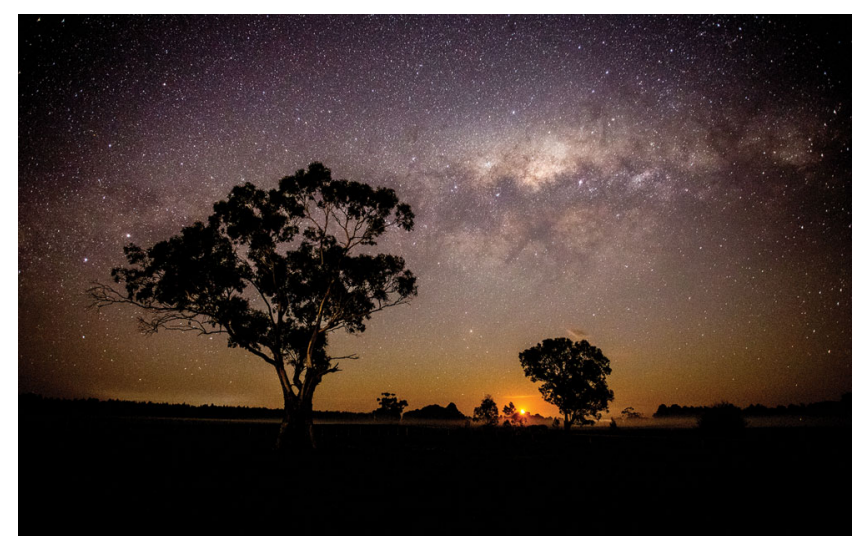

FIG. 3. Image of the night sky showing the Milky Way galaxy. Image credit: Saeed Salimpour. 


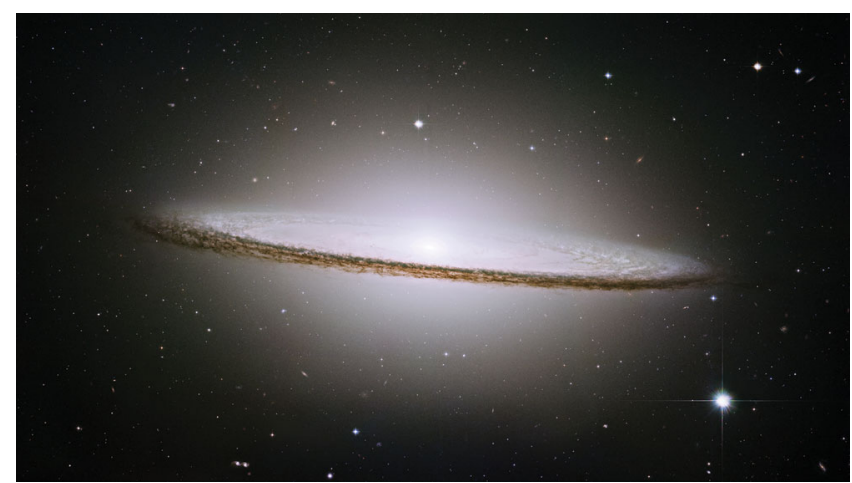

FIG. 4. Image of Sombrero Galaxy (M104), a lenticular galaxy located around 29 million light years away. Our understanding of cosmology and the scale of the Universe drastically altered when it was determined that objects (galaxies) like M104 were objects (galaxies) like the Milky Way, and not structures within the Milky Way itself. Image credit: HST/ESA/STScI.

astronomical objects and their associated spatial and temporal relations beyond the Milky Way galaxy.

The image of the galaxy NGC4526 showing the supernova (an exploding star) SN1994D (Fig. 5) represents an important method in astronomy. By measuring the brightness of these special types of supernovae (type Ia), astronomers can measure distances to distant galaxies. Here, the disciplinary aesthetic involves an appreciation of these practices associated with the image on two levels:

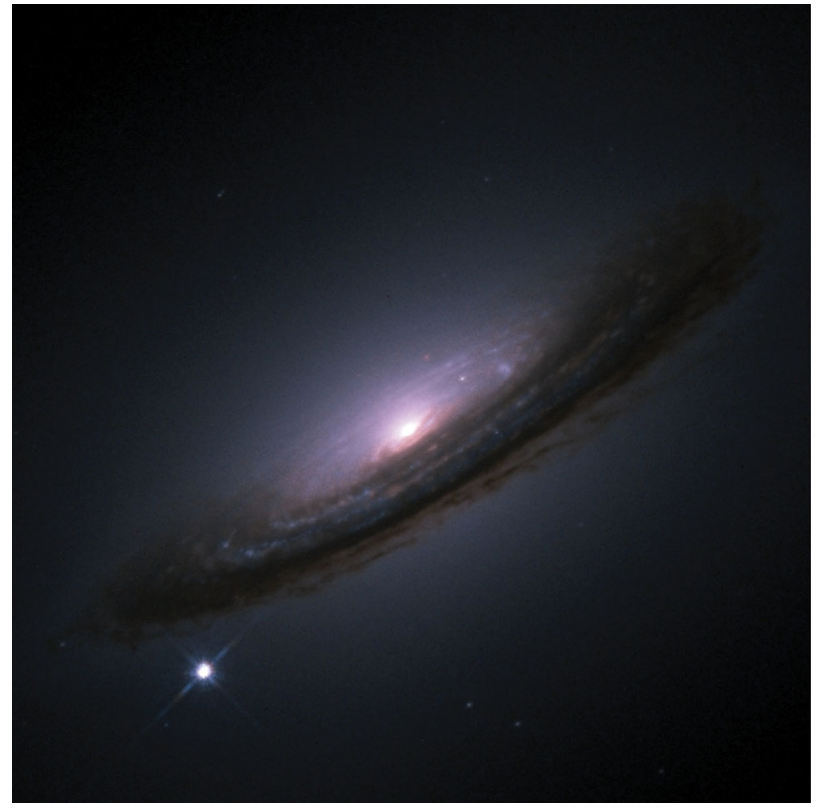

FIG. 5. Image of the galaxy NGC 4526, showing SN1994D (SN short for Supernova). Type 1a supernovae, which are a particular class of exploding stars, are key to measuring extreme distances in cosmology. They were also key in working out that the Universe on the large scale is undergoing accelerated expansion. Image credit: NASA/ESA, The Hubble Key Project Team and The High-Z Supernova Search Team. (a) concerning the information that can be extracted from an image; (b) being able to capture an image of a star that has exploded. Galaxies are key components in understanding the Universe, because they are an important part of the large-scale structure of the Universe.

Figure 6 extends our horizon and understanding as it represents a key advancement in cosmology. The Hubble Ultra Deep Field (HUDF) is an image that has a field of view of about one-tenth the angular diameter of the full Moon. The resulting image shows an estimated 10000 galaxies. Some of these galaxies are from a time when the Universe was only a few hundred million years old. Alongside the everyday and art aesthetic, the disciplinary aesthetic represents an appreciation of the narrative of discovery, including the unexpected and surprising nature of phenomena in the Universe.

The interplay between art aesthetic and disciplinary aesthetic associated with the interpretation and appreciation of such images is seen in the work of the world-renowned astronomer and astronomical imaging specialist David Malin (Fig. 7). In Fig. 7 one can appreciate the art aesthetic associated with the striking color palette that is surreal, and the composition of the image. However, a scientific disciplinary aesthetic involves appreciation of the technicalities associated with capturing the image and the careful

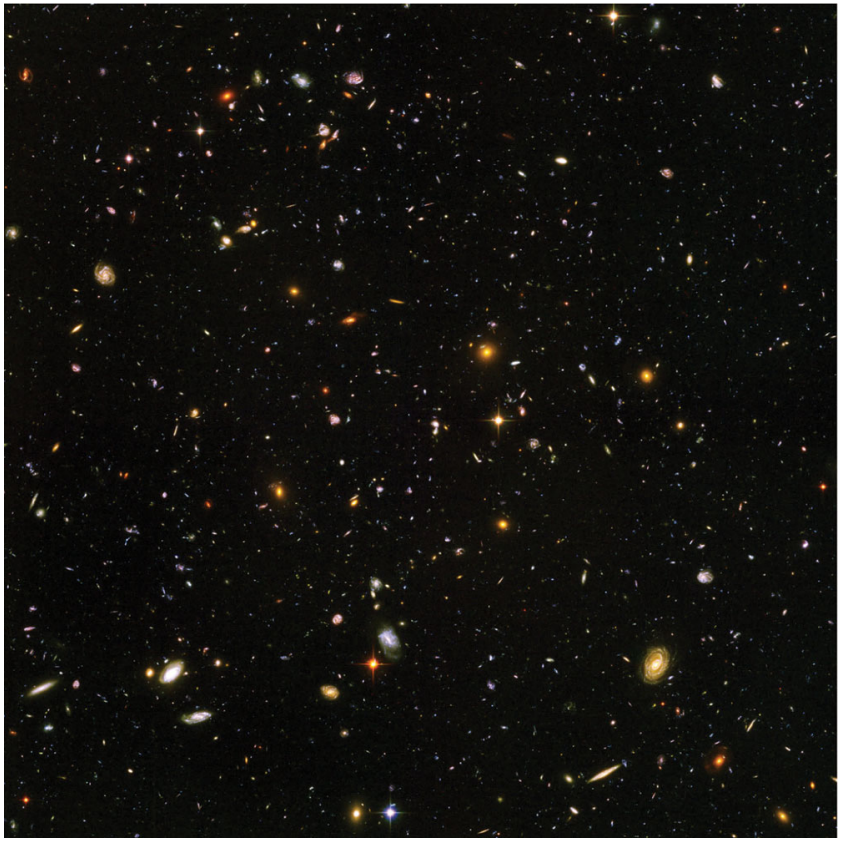

FIG. 6. Image of the Hubble Ultra Deep Field (HUDF). This image is a sight to behold, covering a patch of sky about $1 / 10$ the angular diameter of the full Moon. The angular diameter of the full Moon is $1 / 2$ degree. The image contains an estimated 10000 galaxies, in a patch of the sky that appeared "empty." Images like the HUDF are vital in providing a comparison of the evolution of galaxies in the early Universe (high-redshift) to galaxies as they are "now" (low redshift) like M104 in Fig. 4. Image credit: NASA, ESA, and S. Beckwith (STScI) and the HUDF Team. 


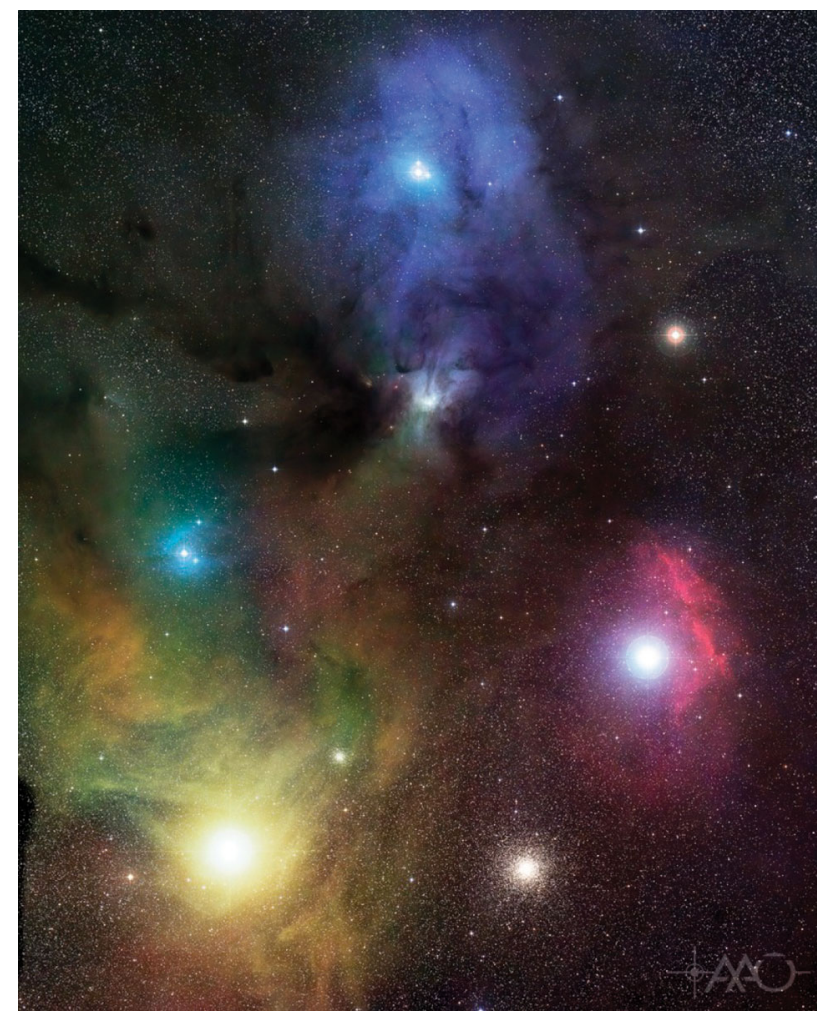

FIG. 7. Antares and the Rho Ophiuchi dark cloud. One of the early works of David Malin, combining black and white astronomical images (glass negatives), taken in different filters. Image credit: David Malin/AAO/UKS (1979).

use of representational color, to highlight the different features and physical processes at work [67-70].

Below we offer a narrative to illustrate the interlocking art and disciplinary aesthetics associated with the interpretation and appreciation of such images. For the first time the extensive curated exhibition of David Malin's photographs-Night Skies: The Art of Deep Space, highlighted the synergy between art and disciplinary aesthetics. In the exhibition book, the curators Eileen Slarke and David Malin, each provided a commentary on selected photographs [75]. David provided the commentary from a scientific lens, which included the disciplinary aesthetics, explaining the science behind not only the image, but also the physical mechanisms operating in the astronomical object and its environment. David provided this commentary using a rich, poetic language- - The dust is also evident in silhouette, both as sinuous dark lanes winding through the luminous gas" (p. 28), or "The soft red glow of the fluorescent hydrogen is evidence that there are young hot stars associated with the dusty clouds" (p. 28). Here we see the presence of disciplinary aesthetic, where David communicates not only the disciplinary affordances associated with astronomical conceptual entities, but also the aesthetic aspects of these representations that show the close interweaving of meaning and feeling. Eileen's commentary used a visual arts lens; comparing the images to styles of visual arts and artists: "Although this image belongs to Gauguin in colour, in technique it belongs to the 'pointillists', especially the work of Paul Signac and his Portrait of Felix Fénéon." There is a complex relationship evidenced here, between disciplinary and art aesthetics.

Astronomers creating representations (color images of various aspects of the Universe and objects within it, models, data visualizations) make both disciplinary and art-aesthetic decisions, for example, the color palette to use and what color to allocate to a particular filter $[67,69,70]$. They also aim to preserve a certain awe (everyday aesthetic) associated with the Universe, in addition to representing the physical and chemical processes taking place. In representations involving color imagery there is strong harmony between art and science. There is a tight binding, particularly in these photographic images, between disciplinary conceptual knowledge and disciplinary, art, and everyday aesthetics.

In summary, aesthetic responses are an important aspect of conceptual learning $[49,74]$ and a serious analysis of representations used in astronomy or cosmology needs to include aesthetic features. We have argued that three types of aesthetic (everyday, art, and disciplinary) are prominent in representations in this discipline, the interactions of which can provide scaffolding in the learning process. From a pragmatist perspective, aesthetic responses are tightly bound to conceptual understanding, and everyday and art aesthetic provides the scaffolding to guide students to recognizing the disciplinary aesthetic, and the associated disciplinary concepts encompassed by a representation.

\section{Analogies}

Both in disciplinary and in educational discourse analogies are commonly used devices to establish and communicate knowledge in science [58,76,77]. The aim of analogies is to use familiar scenarios to help make tangible the counterintuitive and intangible [78-80]. There is much debate with regards to the effectiveness of analogies and most importantly their effective use in science teaching $[81,82]$; however, there is a widespread use of analogies in science teaching. From the perspective of this study, one of the interesting aspects that warrants exploration is the move from analogy in the form of verbal or textual description to one of multimodal representation. It is true that analogies do have visual forms; however, those forms often manifest as the analogy is unpacked. For example, a teacher explaining the concept of the expansion of the Universe may start by saying "Imagine a balloon with dots on it. As the balloon is inflated the space between the dots increases. The dots are the galaxies". The teacher may then decide to get a balloon and demonstrate this scenario. This is a common analogy used in cosmology to help visualize the large range of scales involving galaxies and the Universe by bringing these to a human scale. 
To unpack analogies in cosmology this study uses the theoretical perspectives of structure mapping [50]. Structure mapping in essence aims to highlight the relationships between the parts of an analogy that unpack conceptual structure, rather than literal similarities. Using structure mapping allows us to unpack whether the relationship between the parts is accurately conveying the underlying concepts. This is important as it has implications for whether an analogy creates alternative conceptions that are not aligned to the intended aim of the analogy.

\section{RESEARCH AIM}

This study reviews and analyses a variety of representations in cosmology that serve disciplinary and/or pedagogical purposes. Its aim is to develop a framework that can make sense of the meaning-making processes that underpin the interpretation of these representations, which will provide a guide for teachers and their students to unpack these representations. The theories highlighted above provide a foundation for the framework through informing the exploration of cosmological representations.

Using the conceptual framework illustrated in Fig. 2, this study aims to explore the following research questions:

- What does the landscape of cosmology representations look like for different audiences and different conceptual dimensions?

- How can we systematically describe the varying nature and purpose of representations in cosmology education?

- How is the translation made between disciplinary representations and representations in cosmology education?

\section{METHODOLOGY}

The methodological approach for this qualitative study is situated within grounded theory [83,84] and multimodal analysis (e.g., Refs. $[41,85,86])$. Qualitative data analysis is an inductive approach that looks for patterns and relationships. This study uses a subset of qualitative analysisthematic analysis [87] — with the aim of extracting meaning and patterns from a range of representations, in order to develop a theoretical framework.

The analysis in this study concerns the structures of multimodal semiotic resources, drawing on the theoretical perspectives of Kress, van Leeuwen, O'Halloran, and Jewitt $[41,85,86,88]$. Representations possess a certain "visual grammar" [6]; the components of which interact to create meaning as a whole-gestalt. This visual grammar is also discipline specific, and as such a level of disciplinary knowledge is required in order to extract meaning.

The aim of this study is to use qualitative data to develop a framework for analyzing representations. The methodological approach is best described by grounded theory $[83,84]$, which has some features that are suited to this work. These include being inductive, comparative, interactive, iterative and abductive. Abduction or abductive reasoning is a process of inference making, the others being deduction and induction [89]. "Deduction proves that something must be, Induction shows that something actually is operative, Abduction merely suggests that something may be." (Ref. [90], p. 216). Peirce defines abduction as the "process of forming an explanatory hypothesis" (p. 216). The methodological approach makes it flexible and responsive to the anomalies and surprising findings that may exist in the data. The researcher is then tasked with suggesting possible explanations for these anomalies.

Within grounded theory a process of coding called constant comparative method [83] was used. The key feature of this method is that the various units of data are analyzed and compared with other units. This process allows for the comparison, organization, and synthesis of the similarities, differences, and concepts present within the range of representations in cosmology. The process is iterative, whereby each finding is incorporated into the process of analysis and guides the refinement of codes as they are developed. Every step of the analysis required the consideration of the analysis of previous units to inform and guide the proceeding steps. The process was nonlinear and required the researchers to be receptive to the information contained within the data. The dataset was chosen to represent the rich variety of cosmological representations dealing with a range of aspects of cosmology concepts and their evidence base.

The first step involved an exploratory search to identify the various representations that are canonical in cosmology. This was achieved by using keyword or phrase searches in the Astrophysics Data System, Google, and a search through textbooks at various education levels. The keywords were based on key concepts in cosmology. These were derived from curriculum statements at the secondary school level, textbooks at the undergraduate level, popular science textbooks, and drawing on the authors' background in the field. Some of the keywords included but were not limited to the following: evolution of the Universe, shape of the Universe, dark matter, epoch of reionization, curvature of the Universe, cosmological models, expansion, redshift, redshift surveys, large-scale structure, CMB. The representations selected were based on those that are most prevalent in cosmology, and/or those that are used to communicate key concepts in cosmology. The identified representations were then grouped into four main conceptual dimensions relevant to cosmology based on previous work by Salimpour et al. [91]: size and scale, spacetime location, composition of the Universe, and evolution of the Universe. The representations were also grouped based on the mode (video, images, simulations, interactives), and the education level (high school, undergraduate, post-graduate, public outreach, and professional) at which they appear. 
Following this a preliminary coding scheme was used to highlight the different aspects of each representation, for example, presence of text, use of notations. This was merely a categorization to identify commonalities among representations. As more representations were found the coding scheme grew. Next those representations that had variations were examined, and the manner in which the representation had evolved was coded. "Evolved" refers to how a particular representation had been modified to be used for different educational or communicative purposes, and/or had changed over time. The final stage looked at the representations to describe the sort of knowledge they were aimed at communicating and also the knowledge required by the reader of the representation. This process allowed for the identification of commonalities among representations. The various codes were combined into themes [92-94], which provided the basis for the development of a framework.

To ensure both inter- and intracoder agreement [95] the above process was carried out by the members of the research group and by the first author at different times. The authors also held meetings focused on the analysis during the refinement and grouping of the codes. The unit of analysis was a single representation. An open coding scheme was used, whereby the codes were developed as a result of the analysis by the authors. This ensured flexibility and provided a more robust discussion in the analysis meetings.

\section{DISTRIBUTION OF REPRESENTATIONS}

In order to understand the landscape of cosmology representations we examined not only the representations as such, but their distribution across the conceptual dimensions of cosmology [91], and also the different levels [public outreach (PO), high school (HS), undergraduate (UG), postgraduate (PG), and professional (PRO)], to provide insight into the relative prevalence of cosmological ideas for these different audiences.

Overall, over 200 representations, including variations in the same type of representation were examined from textbooks (high school, undergraduate, postgraduate), journal articles, organizational websites (e.g., NASA, ESA, ESO), and research collaborations (e.g., Illustris, 2dF Redshift Survey, SDSS). This is visualized in Fig. 8 according to the four conceptual dimensions. Some concepts appear in more than one conceptual dimension depending on the particular intent of the representation. For instance, the appearance of "distribution" in both size and scale, and spacetime location, relates to the manner in which the concept of distribution was used in the representations. In the case of size and scale, the distribution of galaxies in redshift surveys was used to convey a sense of scale. In the case of spacetime location, the redshift surveys were used to orient the viewer in terms of relative position. In the case of the $\mathrm{CMB}$ appearing in

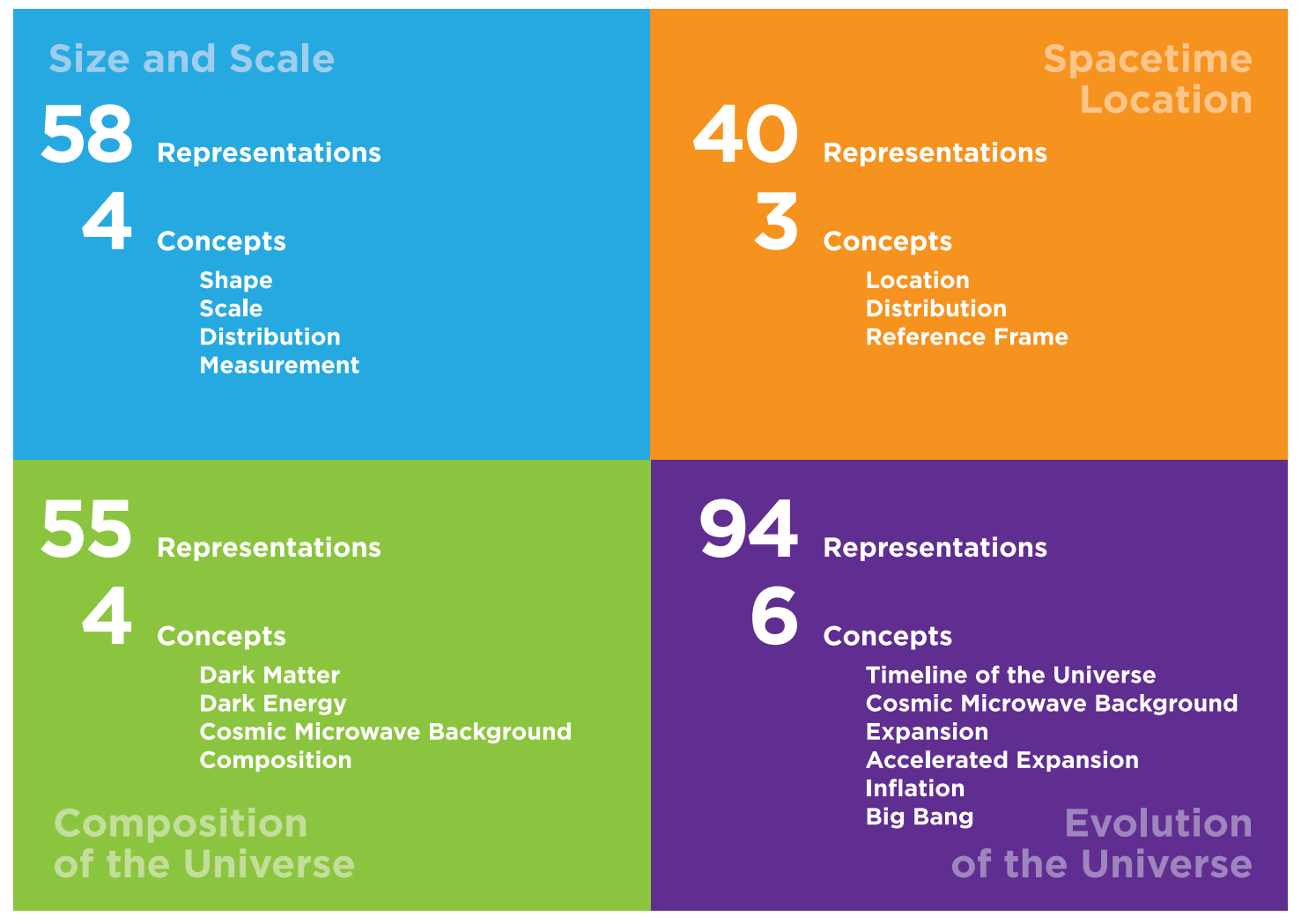

FIG. 8. The landscape of representations in cosmology, showing the number of representations and the various concepts they encompass categorized according to the four dimensions: Size and scale, spacetime location, composition of the Universe, and evolution of the Universe. 


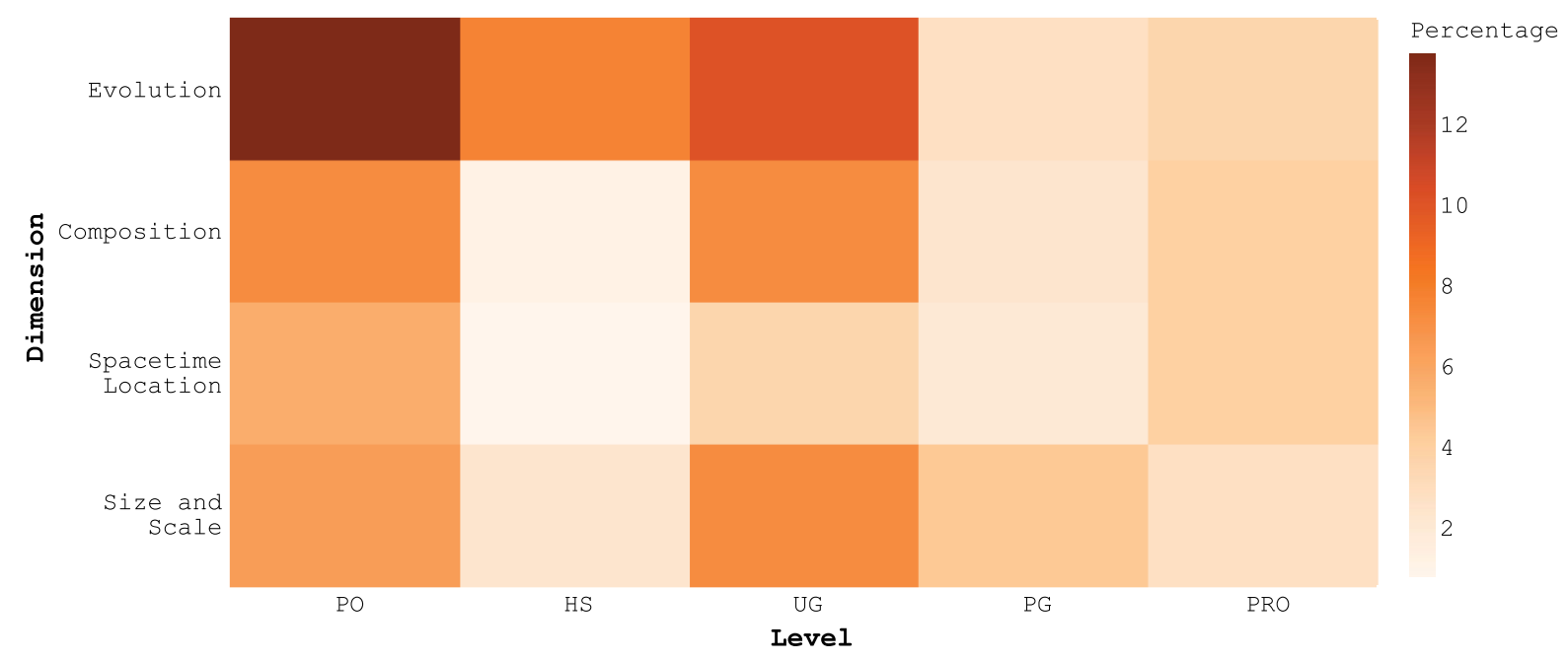

FIG. 9. A visualization showing distribution of the representations for each target audience level (Public outreach, high school, undergraduate, postgraduate, and professional) across the four dimensions, as a percentage of the total number of representations $n=247$. The darker shades represent higher percentages.

both composition and evolution, this was distinguished based on whether the CMB was used to communicate concepts about the composition of the Universe or if it was used to support understanding the evolutionary history of the Universe. The categorization was based on iterative runs by the authors of the study and focus meetings.

To understand how the various conceptual dimensions were distributed across the various educational audiences the data was used to generate Fig. 9. It can be seen that compared to the evolution of the Universe; the other three conceptual dimensions do not have as many representations aimed at high school, and these are often taken from public outreach or undergraduate level.

Using the curriculum statements and chapters from various textbooks key concepts were extracted, for example, expansion, dark matter, and CMB. These concepts in cosmology had various representations associated with them. Figure 10 presents the distribution of concepts for each of the five levels (PO, HS, UG, PG, PRO). Some of the insights from this analysis are as follows:

- The seemingly low number of representations aimed at and developed specifically for HS level.

- The preponderance of timeline (showing the evolutionary history of the Universe) representations for PO.

- The differential patterns of focus at different levels, for instance, representations related to mapping are strong in PO and PRO level but not in HS or UG. This is because mapping representations are directly based on observational data, which is produced

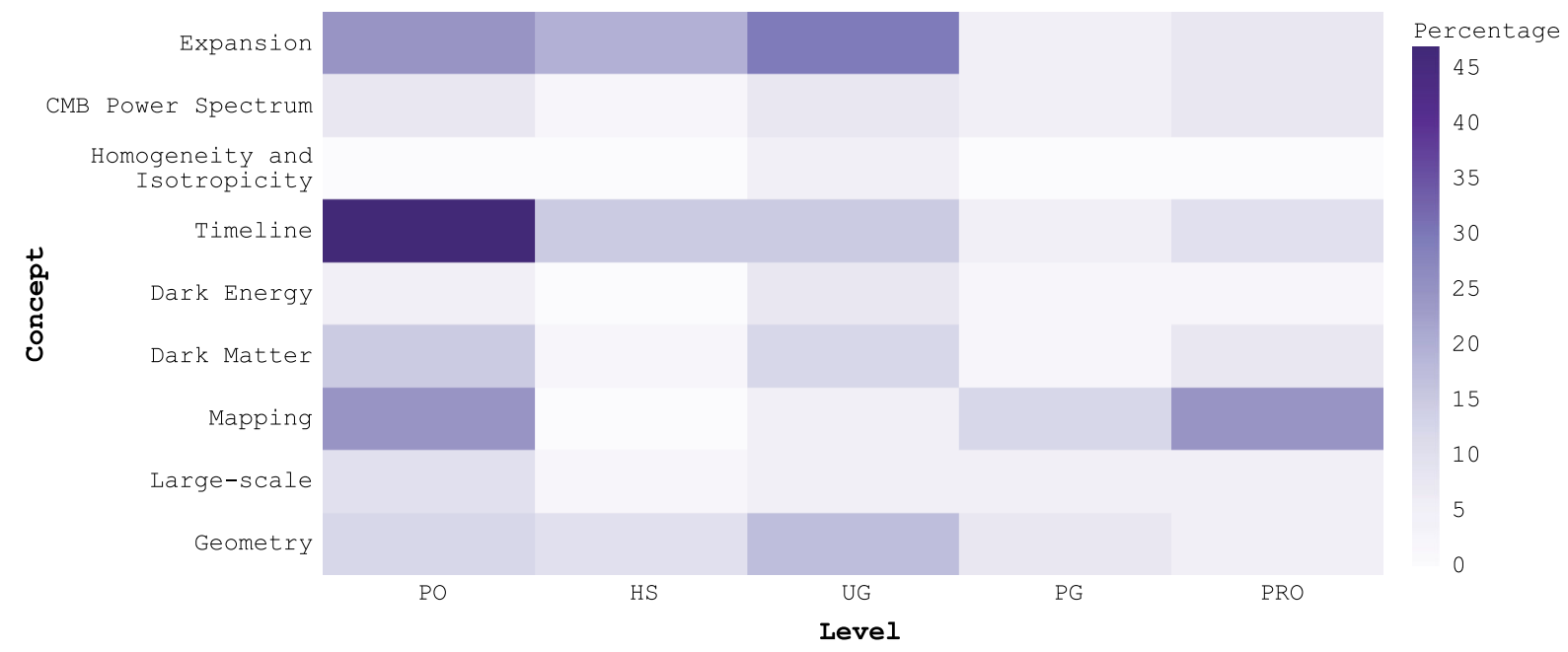

FIG. 10. A visualization showing the distribution of representations communicating specific concepts in cosmology at each of the target audience levels (public outreach, high school, undergraduate, postgraduate, and professional), as a percentage of the total number of representations $n=247$. The darker shades represent higher percentages. 


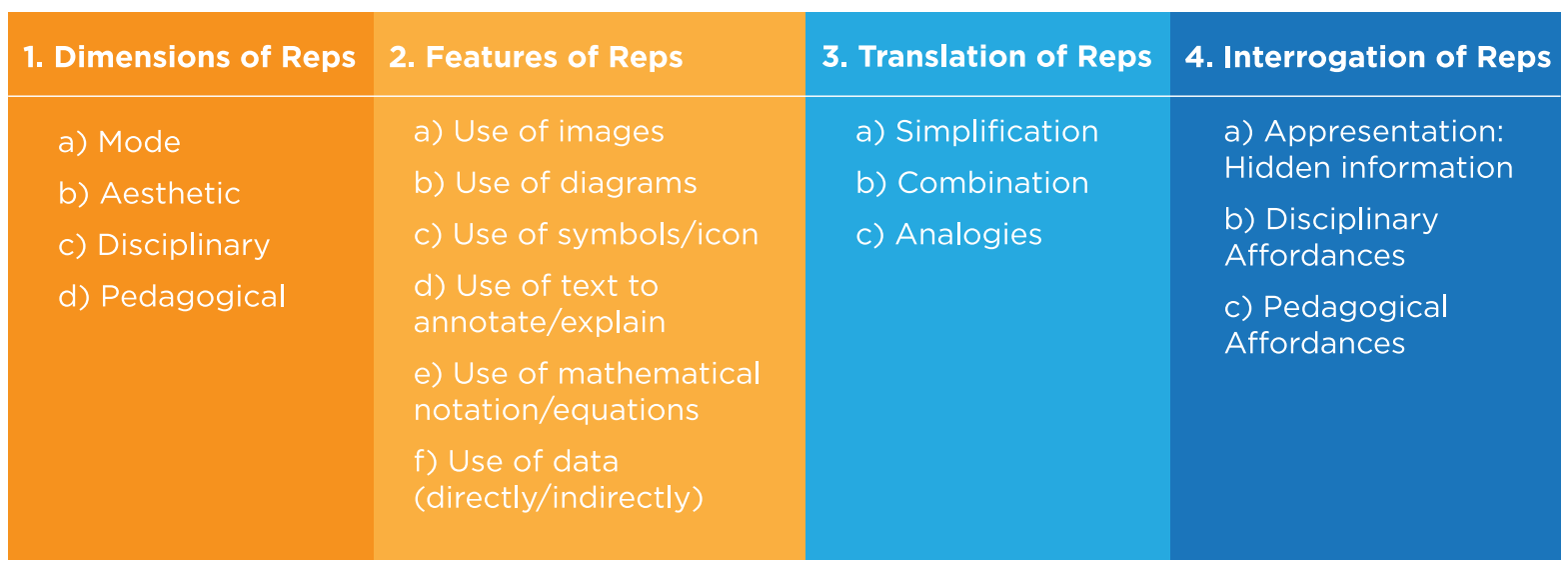

FIG. 11. Characteristics of representations ("Reps").

primarily at the PRO level, and often used to communicate to the public.

The aim was to find representations that addressed concepts in cosmology curricula. This aim set a boundary condition for the sampling of representations. As such, it can be noted that the number of representations at HS, PG, and PRO levels is less overall. In addition, the representations created at a PRO level, and published in peerreviewed journals are mostly in the form of graphs that analyze observational or theoretical datasets. Furthermore, cosmology as a precision observational science is recent; therefore, the number of key concepts that traverse target audience levels is consequentially limited.

\section{DEVELOPING THE ANATOMY OF REPRESENTATIONS}

Using the analysis of the range of canonical representations via the process discussed in Sec. V and drawing on the categorizations of Harrison and Treagust [58], and Gilbert [59], we identified various features that seemed to be consistent and fundamental across representations. These features were coalesced into four overarching categories, with associated subcategories presented in Fig. 11. This category or subcategory structure was built from the iterative coding and grouping process by the authors. The first two categories (dimensions and features) are a categorization scaffold and are highlighted by the orange palette. The next two (translation and interrogation) are where deeper exploration of the representation occurs and are highlighted by the blue palette. Here the reader delves into the semiotic affordances of the representation. Using the categorization in Fig. 11, an initial run over a range of representations from each of the four dimensions (Fig. 8) was implemented to develop explanations for each of the subcategories.

Using the characterizations shown in Table I, the descriptions were synthesized into overarching lines of inquiry in the form of single questions (Fig. 12). Together with the explanatory Table I it is envisioned that teachers can use this framework within their unit of study.
This can be for either a single concept or multiple concepts. The framework proposed is referred to as the anatomy of representations (AOR). The AOR is about understanding the variety of representations that are used in a field, and how the more technical and abstracted disciplinary representations are transformed for communication purposes. Of course, this communication might be for other disciplinary experts, but often they are for the public and educative purposes. As a second purpose, understanding the nature of the representations and how they operate to make disciplinary representations understandable for students is potentially very useful for teachers and other educators such as textbook authors, and science communicators generally.

Taking guidance from the manner in which representations were interrogated during the analysis, a synthesis of the categories into four encompassing layers was made (Fig. 12). Within each layer the reader of the representation starts by asking a single guiding question as shown in Fig. 12. As the reader peels back each layer going from the outermost layer to the core, they discover characteristics of the representation and the devices used to help with the reading of the representations. This framework is designed to be a guide when unpacking representations in the context of teaching and learning. The fundamental idea is that every representation is composed of layers. To understand a representation, its purpose, evolution, and most importantly the reading of the representation requires the peeling back of these layers. Evolution in this case refers to the representation changing over time, across modes, or target levels. The rationale for the layered approach is twofold: (i) it allows the steps to be chunked so that it is manageable in a classroom setting; (ii) to delve deeper into representations it is necessary to understand the categorization first, because that information can help guide the interrogation of the representation.

\section{A. Meaning making in representations}

In the AOR framework the two innermost layers "translation of representations" and "interrogation of representations" draw on well-established theoretical and empirical 
TABLE I. Explanation of the various subcategories.

1. Dimension of representations: This category relates to the format of the representation and its purposes in relation to audience. Subcategory Description

(a) Mode

(b) Aesthetic

This relates to whether the representation is an image, video, simulation or interactive. This is flexible as more categories can be added; for example, image can be a drawing, diagram, photograph etc.

This relates to how aesthetic elements and decisions have been implemented in the representation. The aesthetic also relates to the aesthetic experiences a reader of the representation may have. This is not only in the "eye of the beholder," but rather specific features also associated with visual design principles. This encapsulates the everyday, art-aesthetic and disciplinary aesthetic.

(c) Disciplinary This relates to whether the goal of the representation is disciplinary or not. For example, it uses conventions accepted in the disciplinary community.

(d) Pedagogical This relates to whether the goal of the representation is to teach concepts to various levels. Pedagogical representations are designed specifically for pedagogical purposes as a variation on the full disciplinary representation. They do not always require disciplinary knowledge, and often the requirement is at a lower level than that of a disciplinary representation. This sub-category describes the particular features that are different to or allow for the unpacking of the full disciplinary representation features with a pedagogical aim.

2. Features of representations: This category relates to components that constitute a representation. Representations can be composites made up of various semiotic systems and resources.

Subcategory

(a) Use of images

(b) Use of diagrams

(c) Use of symbols or icon

(d) Use of text to annotate or explain

(e) Use of mathematical notation or equations

(f) Use of data (directly or indirectly)

\section{Description}

This relates to whether the representation uses imagery; for example, photographs of galaxies. This relates to whether the representation incorporates diagrams as part of the composition. This relates to whether the representation uses symbols and icons. These could be pedagogical or disciplinary.

This relates to whether the representation uses text to communicate hidden information in the representation; or to clarify the information presented in the representation.

This relates to whether the representation uses mathematical symbolism to help communicate information that would not be possible via text. These can be at different levels of complexity or sophistication depending on intended audience.

This relates to whether the representation uses data, whether it is directly in the form of a graph; or indirectly of an image based on data; for example, the cosmic microwave background map.

3. Translation of representations: This category relates to how representations change or are repurposed for a particular context. Subcategory Description

(a) Simplification This relates to how the representation has been changed to change the affordances. This change could be from the full disciplinary representation to make it accessible at a particular level. This can either by via the addition or removal of elements or semiotic resources.

(b) Combination

(c) Analogies

This relates to how the representation uses a combination of one or more separate representations. This relates to the representation of analogies for pedagogical purposes. Analogies can be simplifications. However, simplifications can happen without analogies, for example, taking a graph and removing the disciplinary aspects.

Interrogation of representations: This category relates to a deeper exploration of representations, which include the aims and the meanings they encompass for various audiences.

\begin{tabular}{|c|c|}
\hline Subcategory & Description \\
\hline (a) Appresentation & $\begin{array}{c}\text { This relates to the hidden information that the representation contains but is not made explicit. Often } \\
\text { appresented information is only accessible at a high degree of disciplinary discernment. }\end{array}$ \\
\hline $\begin{array}{l}\text { (b) Disciplinary } \\
\text { affordances }\end{array}$ & $\begin{array}{l}\text { This relates to the degree of disciplinary information that is contained in the representation. It is directly related } \\
\text { to the concept that the representation frames and enables. The disciplinary aesthetics of the representation } \\
\text { can be further unpacked here. }\end{array}$ \\
\hline $\begin{array}{l}\text { (c) Pedagogical } \\
\text { affordances }\end{array}$ & $\begin{array}{l}\text { This relates to the degree of pedagogical scaffolds that are contained in the representation. In essence, how the } \\
\text { representation scaffolds conceptual understanding for the students. It also depends to some extent on how the } \\
\text { educator presents the representation to the student. }\end{array}$ \\
\hline
\end{tabular}




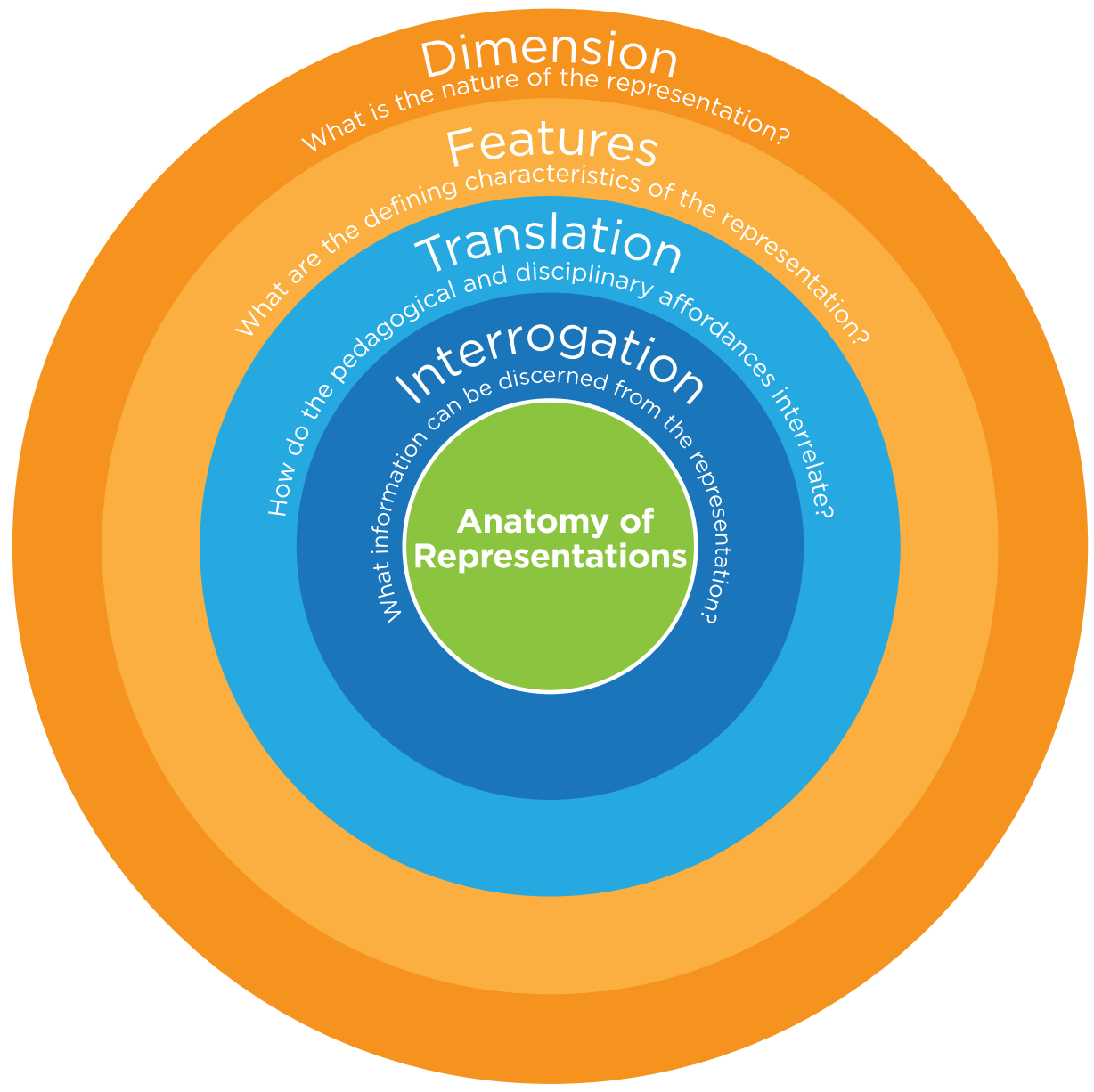

FIG. 12. The anatomy of representations theoretical framework. The reader begins at the outermost layer and gradually works their way to the core. The coloring indicates that the first two layers are related to categorization of representations (oranges), while the inner two layers are a deeper exploration of representations (blues). An interactive version of this is available at https://www .untangleduniverse.com/aor/.

studies in social semiotics, multimodality, and representations [17,41,42,45]. Kress [41] defines an overarching classification translation, which is broadly defined as "a process where meaning is moved." Mode in the context of this study is taken to be a semiotic system; this could be an image, animation, video [96]. A semiotic system consists of a range of semiotic resources that include but are not limited to equations, graphs, symbols, and data.

Kress [41] also defines transduction as a specific type of translation, where "meaning material" is moved from one mode to another; for example, speech to image. Svensson and Eriksson [96] define transduction "as a shift from a semiotic resource to another, but also a shift from a semiotic system to another." (pp. 2-3) Transduction therefore can happen as you align and interpret the different modal images, or in relation to the construction of the representation overall. This can occur through a series of what this current study defines to be translational moves. These moves include:

1. Simplification, whereby the semiotic resources are added, removed, or simplified.
2. Combination, whereby semiotic resources and in some cases semiotic systems are combined.

In the context of disciplinary representations Linder [97] highlights that part of the disciplinary fluency of experts is the ability to discern, notice, and read the hidden information- "seeing what is copresent, or lying behind a representation" (p. 49). Therefore, the process of becoming fluent in disciplinary norms and to be able to read appresented information in representations forms a key part of the interrogation of representations layer in the AOR framework (Fig. 12).

Considering the above, the AOR brings together these concepts to provide a framework for critically analyzing representations found in cosmology.

\section{APPLICATION OF THE ANATOMY OF REPRESENTATIONS FRAMEWORK}

The AOR is a theoretical framework that has been developed by analyzing canonical representations in cosmology. It provides a guide to qualitatively analyze representations. 
The strength of any theoretical framework is determined by its application to practical scenarios. With this in mind we provide examples of the AOR framework in use and discuss the implications for education. We should advise that for practitioners who aim to use the AOR in their classroom, the depth of the interrogation depends on the time available and the key concepts the teacher would like to communicate to the students. This study has focused on images; however, the AOR can be applied to videos, interactives, simulations, and the range of representations present in astronomy.

From the collection of representations two were extracted as examples that were present at various levels (PO, HS, UG, PG, PRO), and also had variations. This allowed the various features of these representations to be unpacked. The two representations concern the concept of geometry of the Universe (Fig. 13), and evolutionary timeline of the Universe (Figs. 14 and 15). Starting with the AOR framework each of these representations is unpacked in Tables II and III.

Drawing on the classification system of Harrison and Treagust [58], and Gilbert [59], it can be seen that Fig. 13 could fit across multiple categories. This highlights the complexity that categorizations are not often mutually exclusive especially when considering representations in

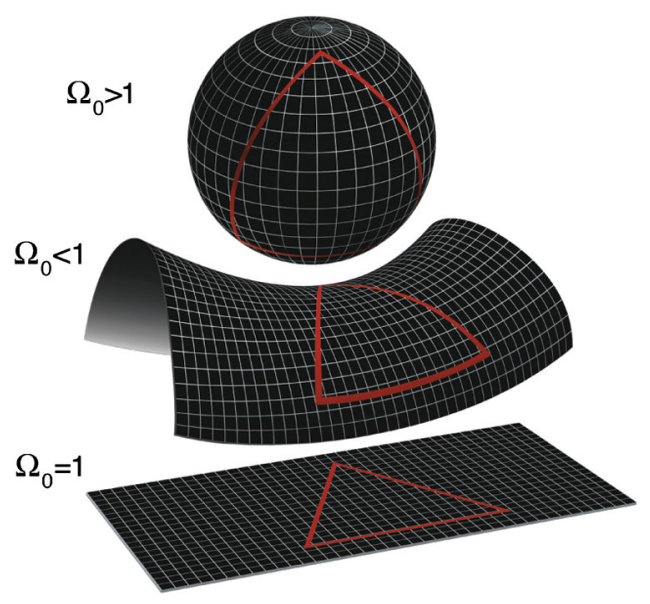

FIG. 13. One variation on the range of representations showing the Geometry of the Universe. Image credit: NASA/WMAP Science Team.

cosmology. Unpacking representations using AOR as shown below allows for the exploration of the multipurpose nature of representations.

\section{A. Geometry of the Universe}

TABLE II. Unpacking the representation of the geometry of the Universe (Fig. 13).

1. Dimension of representation

The mode of this representation is a static image. The art-aesthetic relates to the clarity of the design, employing a classic communication color palette red and black. Red is used to highlight a key concept in this representation - the nature of a triangle on different geometries. Furthermore, we see the use of a grid to help communicate the three-dimensionality of the various geometries. In addition, triangles are distinguished as an overlay to point to a particular feature of the grid - the distortion of Euclidean space means that sides and angles of the triangle are distorted due the curvature of spacetime. The goal of the representation is pedagogical and is used at various levels from high school textbooks to public outreach. However, it requires disciplinary knowledge to read the representation.

2. Features of representation

This representation uses a combination of symbols, and mathematical notation. This representation is supported by a caption that explains what the symbols mean and also the role of the triangle.

3. Translation of representation

This is a classic example of a representation whose variations show simplification and combination. Within the representation itself we are seeing a simplification, where the angles of the triangle are not explicitly shown. The combination is seen in the use of two separate representations: the geometric black shapes and the red triangle. Considering the mathematical notation is itself a representation, we should include that as part of the combination. This representation brings together a number of features with pedagogical intent.

4. Interrogation of representation

This representation requires some disciplinary affordances as there is some hidden information (appresentation). For example, the angles on a triangle add up to $180^{\circ}$ in Euclidean geometry. However, the angles are not explicitly shown they are appresented. The shape of the triangle is a hint to this hidden information. A keen observer or a disciplinary expert would argue that the shape of the triangle is not quite accurate; and in the case of the triangle in the saddle geometry the sides should follow the shape of the saddle. This is perhaps an aspect that the teacher will need to be cognizant of, and use to guide student understanding. Furthermore, the use of the mathematical notation requires the reader of the representation to have disciplinary knowledge of the meaning of Omega $\left(\Omega_{0}\right)$. Interpretation of the representation is challenged by the need to represent 3D geometries-especially distorted spacetime geometries - through a 2D mode. The process of creating such representations has its challenges in deciding what perspective to offer, and student interpretation of this representation needs guidance. 


\section{B. Evolutionary timeline of the Universe}

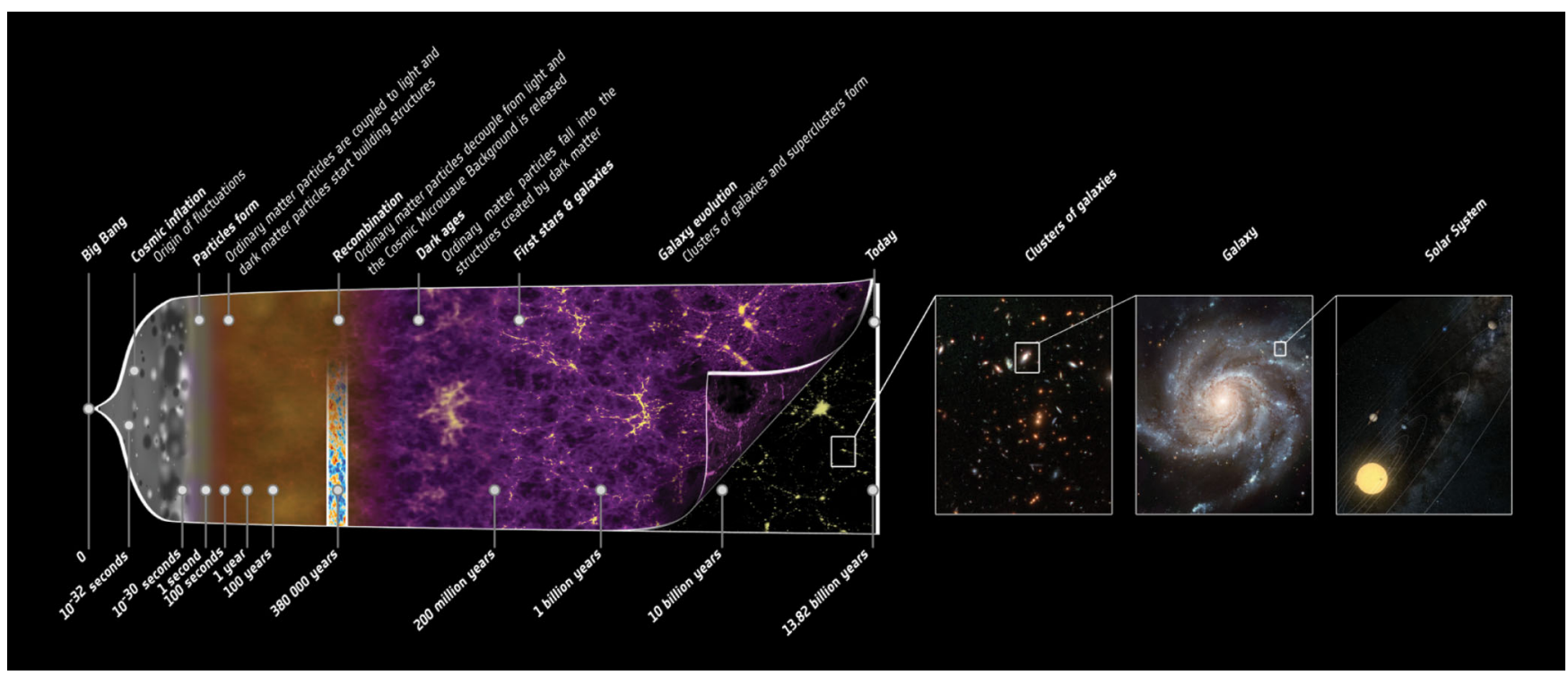

FIG. 14. One variation on the range of representations showing the evolutionary timeline of the Universe. Image credit: NASA/ESA.

\section{$\rightarrow$ COSMIC HISTORY}

\section{Cesa}

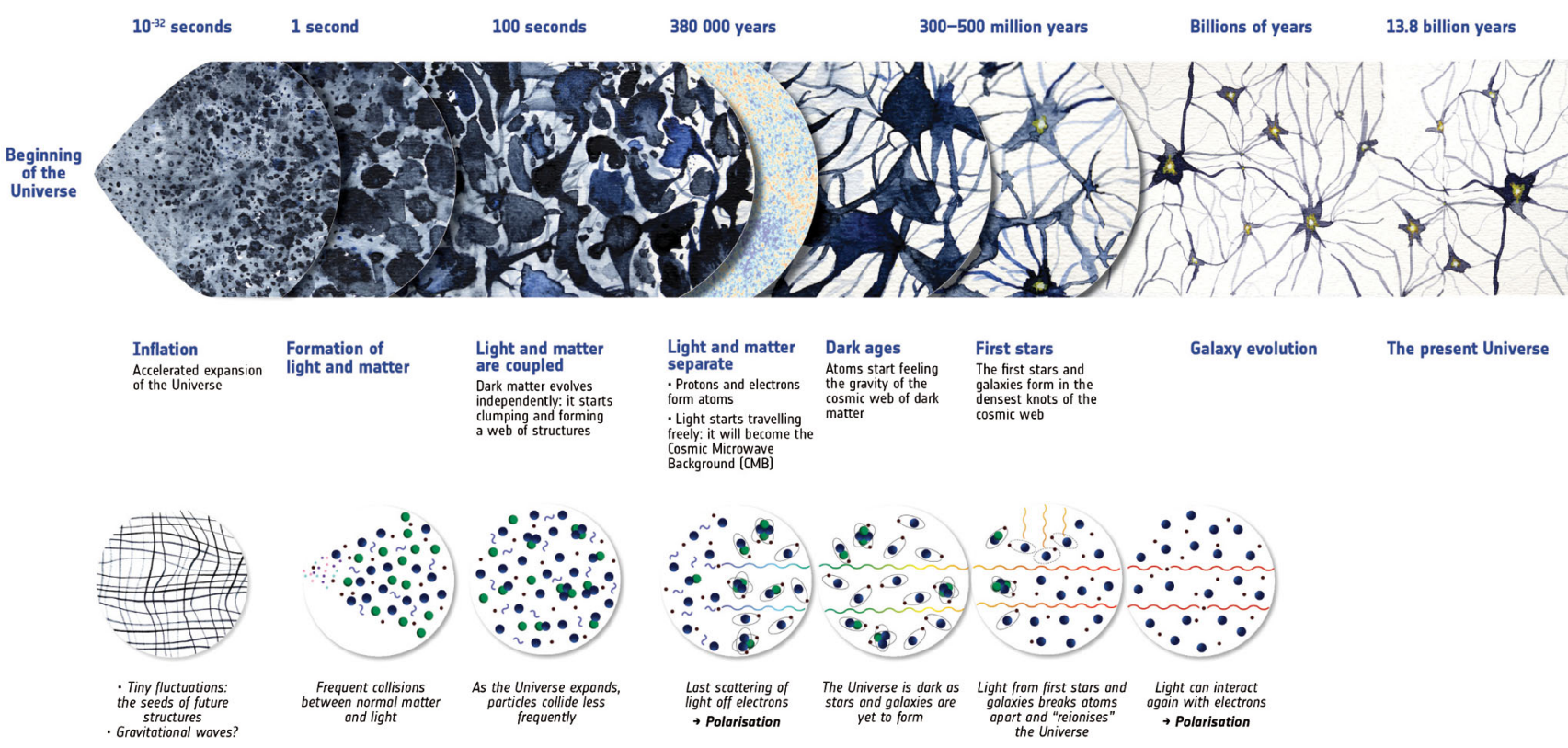

FIG. 15. Another variation of the evolutionary timeline of the Universe. Image credit: ESA. 
TABLE III. Unpacking the representation of the evolutionary timeline of the Universe (Fig. 14).

1. Dimension of representation

The mode of this representation is a static image. In terms of aesthetics, this representation uses strong chromatic contrasts; using color to highlight key events. The representation is primarily pedagogical in nature, owing to the level of information presented and the concepts/research ideas it is communicating. This image is used prolifically in public outreach settings and to a certain degree at conferences to set the scene. The representation with the use of striking images possesses an everyday and art-aesthetic projecting a sense of 'wonder' at the complexity and reach of our Universe.

\section{Features of representation}

This representation uses a mix of images, data driven images, and simulations, which themselves are representations in their own right. It uses simplified text as annotations and there are no mathematical symbols, except for the scientific notation in the numbers.

3. Translation of representation

This representation has a range of variations. However, in the context of this version we can see that it is a combination of various other representations. These include: the CMB which is represented using observational data; the large-scale structure simulation from the Millennium Project; images from space-based telescopes, and a series of computer-generated representations. Comparing this representation with Fig. 15, it has more concise annotations focusing on the key features only.

4. Interrogation of representation

This representation requires disciplinary knowledge to distinguish between real, simulated and illustrative semiotic resources, this information is appresented. This image requires very careful guidance from the educator to highlight some key disciplinary concepts. For example, a student may assume that the "Big Bang" is a single point in space. To appreciate the Big Bang is not a point in space requires the student to achieve a level of disciplinary discernment allowing them to extract this appresented information. The zoomed in boxes are aimed at conveying important concepts: scale, the nature of objects, and how they relate to each other. In a way this provides a scaffolding for students to make connections between these concepts. Furthermore, other features such as the overlay of the dark matter distribution over the luminous matter distribution is an attempt to show the how light does or does not follow mass. Note the use of color to demarcate between dark matter (purple) and ordinary matter (yellow). This becomes important in relation to different models for the evolution of the Universe. The disciplinary aesthetic involves the appreciation of the interplay between simulations and observations.

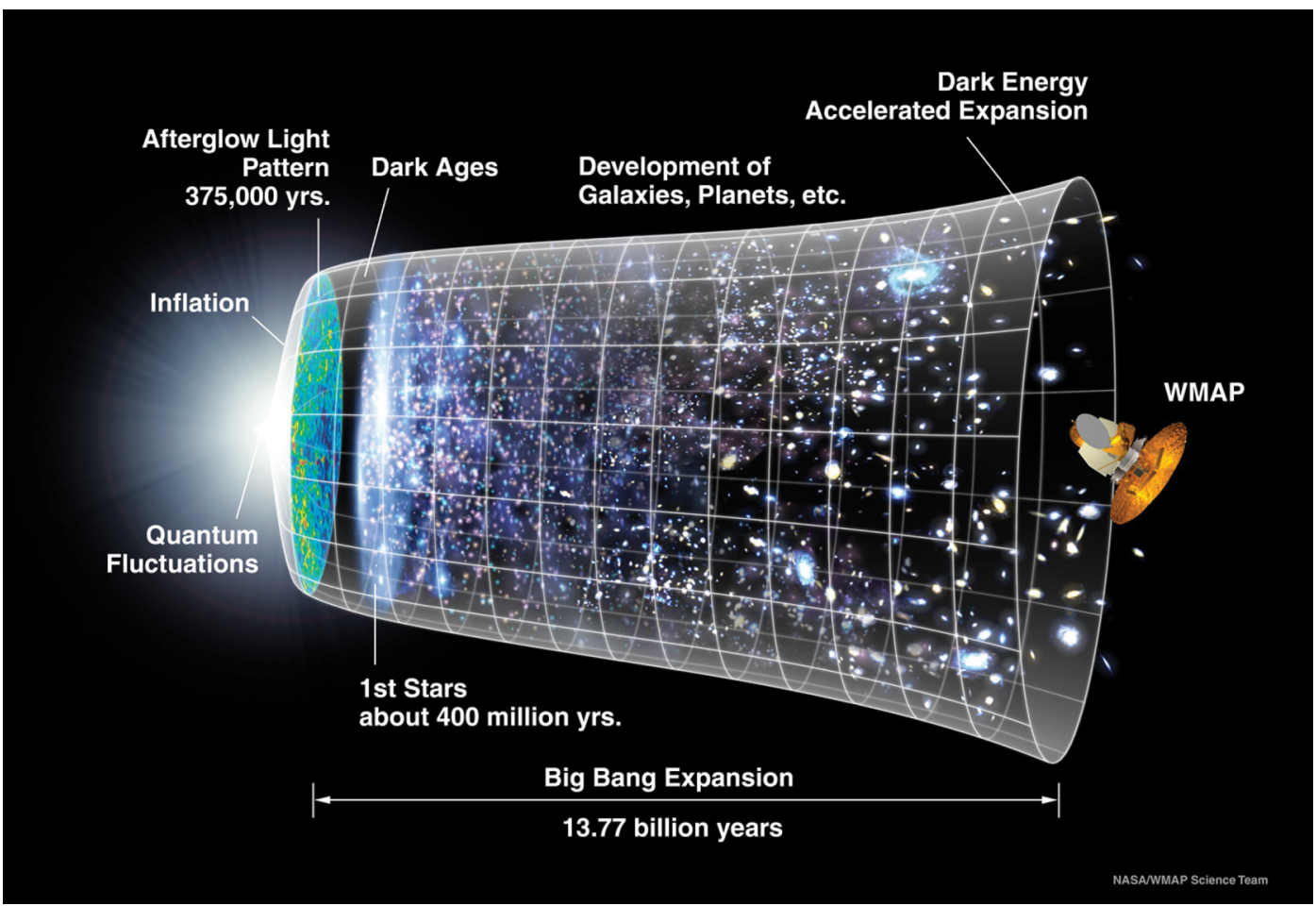

FIG. 16. The timeline of the evolution of the Universe, focusing on the cosmic microwave background and the WMAP satellite which made measurements of the cosmic microwave background. Image credit: NASA/WMAP. 


\section{Variations in evolutionary timeline of the Universe}

The class of representations which communicate the evolutionary history or timeline of the Universe are varied. Two examples are shown in Figs. 14 and 15. Other variations are shown in Figs. 16-18. They are used here to demonstrate the variation in affordances associated with what is or is not shown, and how, as an example of the application of the AOR.

The timeline representation, which aims to the tell the story of the Universe, was found to have more variations than any other. The various versions of this representation each possess different levels of disciplinary and pedagogical affordances, in addition to the aesthetic devices that are embedded in each of the images. These variations include those created by research organizations or universities and also those created by designers for public outreach. The range of semiotic resources within each of these representations provide varying degrees of affordances and are unpacked in Table IV.

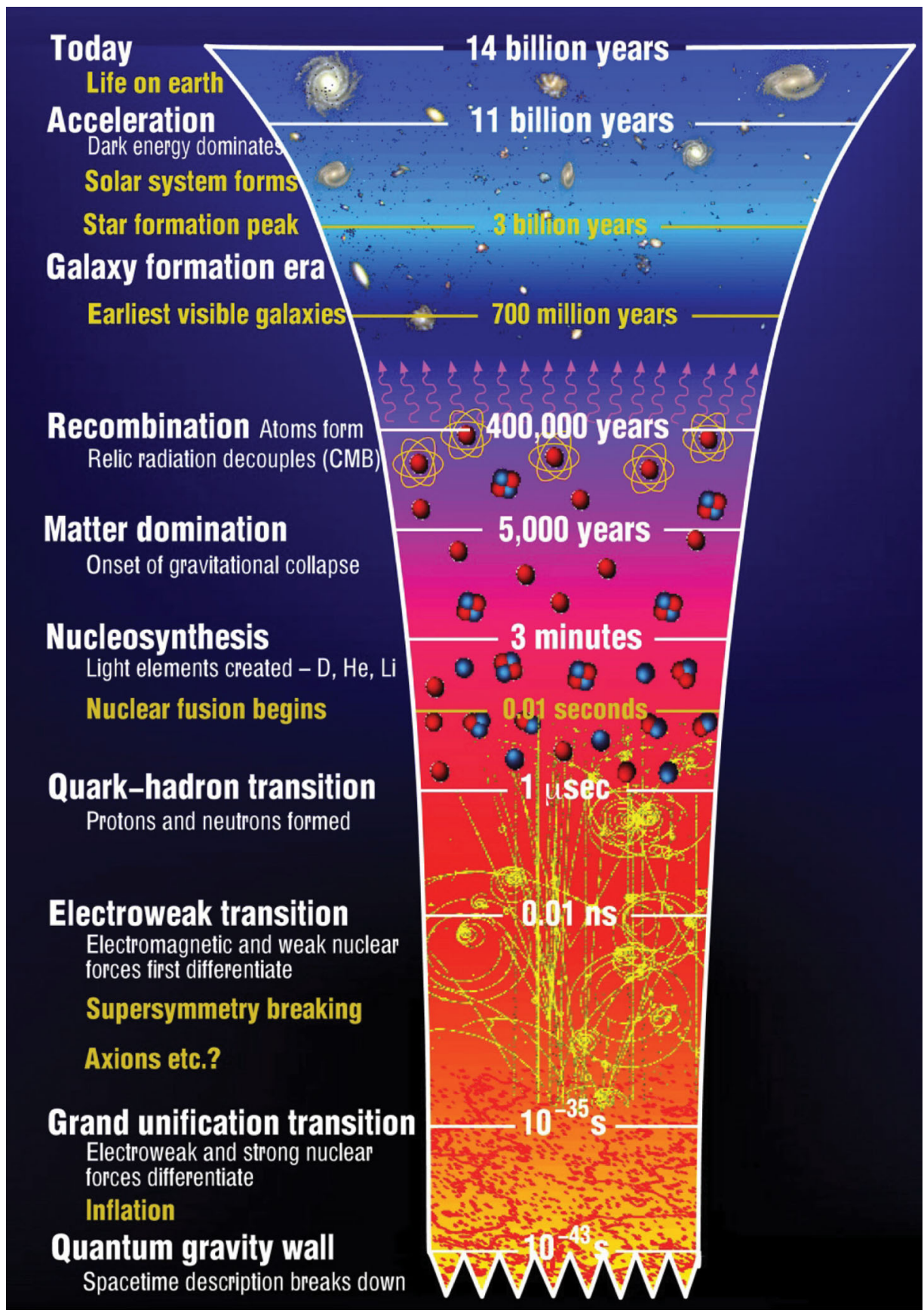

FIG. 17. Timeline of the Universe, another variation of the representations of this concept. Image credit: Center for Theoretical Cosmology. 


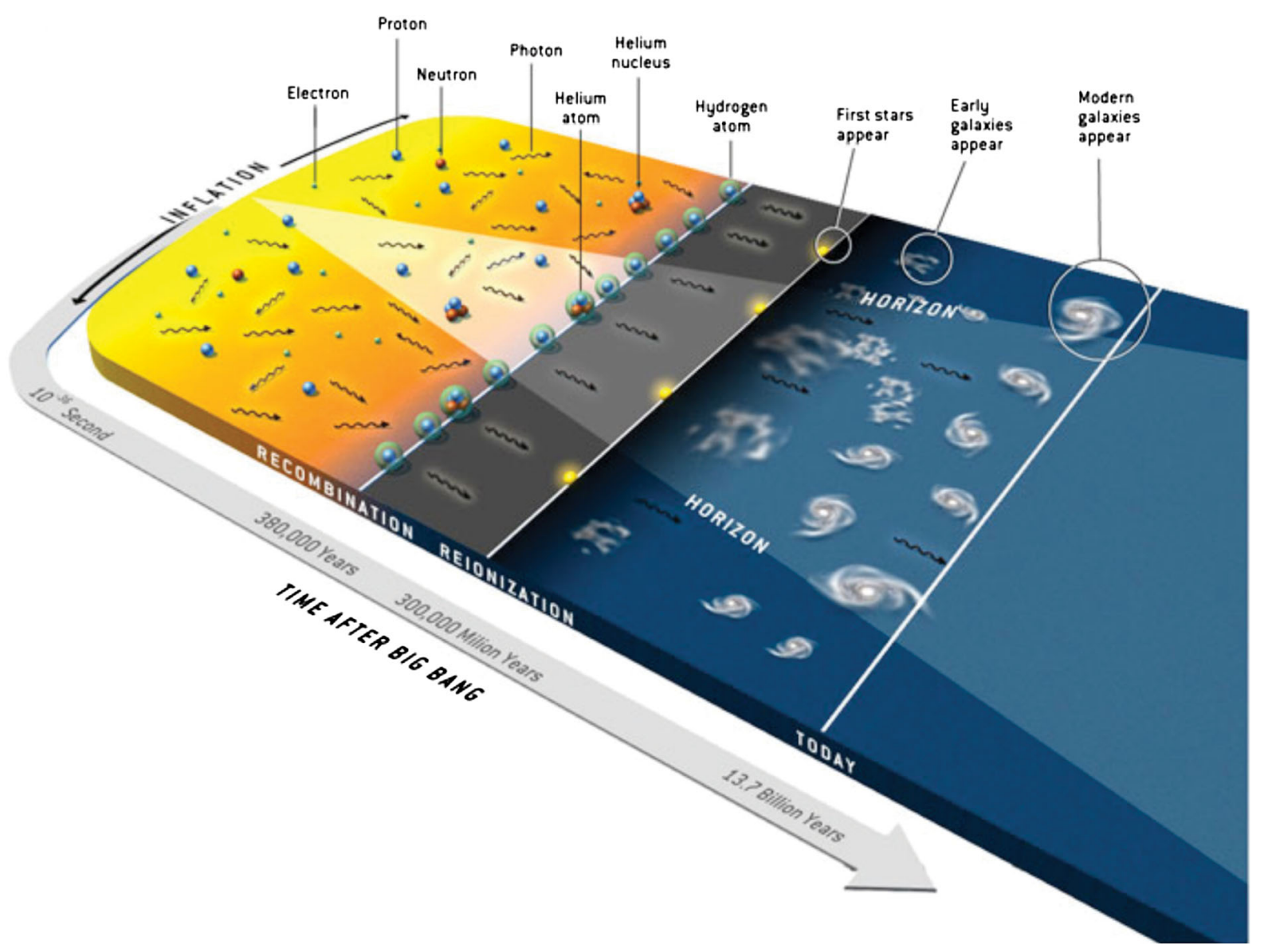

FIG. 18. Timeline of the Universe representation as shown in a popular science article. Image credit: Hu and White (2004).

The AOR framework can be used to compare these representations in a way that highlights the various affordances and the translational moves that exists across the variations (Table IV). This comparison is about determining the features that allow various concepts to be communicated. In addition, we highlight the decisions as to what is foregrounded and what is silenced in the translation from expert knowledge to educational purposes. It demonstrates the challenges associated with rendering a very complex story into pedagogical form. In all the timeline representations, each stage in the evolutionary history of the Universe entails a very dense packing of ideas that carry within them disciplinary knowledge. One aim of the AOR framework is to provide a scaffolding that allows teachers to identify these ideas and help unpack them with their students.

\section{Representations of analogies}

Scientists often use analogies when communicating to the general public, students, and professionally among peers. The use of analogies has a long tradition in science education [76,78,98-100]. This study highlights some analogies used in the form of visual representations designed to have pedagogical affordances. This is distinct from simplification and combination within the AOR framework. There is a growing set of analogies that are used pedagogically to explain the expansion of space, and these have been refined over time. As cosmology matures and its presence in education is established, perhaps we will see a coalescence around analogical representations with well-considered qualities of pedagogical affordance. Analogies by their very nature are limited in the scope of information they can convey. They are designed to simplify a concept, and more importantly help build bridges towards the understanding of concepts. Therefore, a decision needs to be made as to the key information or the theoretical minimum that needs to be maintained to ensure the concept is communicated. A more important decision perhaps is the high-level disciplinary information that is left out, or perhaps simplified, depending on the purpose.

The expanding Universe or more specifically the expansion of space is perhaps one of the key findings in cosmology (ignoring accelerated expansion for the moment as that itself has a dense packing of ideas). One of the most used analogies is that of a bread loaf or cake dough with embedded chocolate bits or raisins. As the dough cooks it expands and so the space between each raisin increases. However, the raisins themselves do not expand. In this analogy, the dough is an analogical representation of the 
TABLE IV. Unpacking the representations of the timeline of the Universe variations (Figs. 14-18).

1. Dimension of representation

The mode of the representations is static image. The art aesthetic with respect to design principles is varied, as each one aims to highlight different ideas. In Fig. 17, the gradation from red to blue is perhaps to highlight the temperature decrease. Keen students would argue that is inaccurate because hotter objects emit at shorter (bluer) wavelengths. The same design principle has been implemented in Fig. 18. This coloration is guided by the everyday experiences and notions of color. Considering Fig. 16, the focus is on the cosmic microwave background, which is highlighted also in Figs. 14 and 15. The everyday aesthetic is encompassed by the striking imagery used in the representations. These representations also possess a disciplinary aesthetic, in their efficient representation of the vast stretches of time and the key stages identified by evolving data instrumentation and analysis. The data used to create parts of the representation includes the cosmic microwave background in Figs. 14-16, or the simulation of large-scale structure via the millennium simulation (purple area) in Fig. 14. This highlights a disciplinary aesthetic also involving an appreciation of the interplay between observation and simulation. Another art-aesthetic design principle is that seen in Figs. 16 and 18, where the Dark Ages era is darkened to communicate the idea that Universe was in fact dark and there were no stars or galaxies. All representations are used in both disciplinary and pedagogical contexts.

\section{Features of representation}

The representations use a mix of semiotic resources: images, data driven images, simulations, artistic renderings, which themselves are representations in their own right. They all use annotations and there are no mathematical symbols except for the scientific notation in the numbers and the disciplinary specific terms especially in Figs. 15, 17, and 18; for example, Quark-Hadron transition, electroweak transition, recombination.

\section{Translation of representation}

Looking at these series of representations, we can see evidence of translation not only within each representation-from data to images, for example with the CMB image (Fig. 16); but also across representations, whereby the addition or removal of various semiotic resources changes the affordance level of each representation. In Fig. 17, for example, we see the increase in disciplinary text, and the use of particle trails between the era $10^{-35} \mathrm{sec}$ and $1 \mu \mathrm{sec}\left(10^{-6} \mathrm{sec}\right)$. Across the representations, some elements of timeline are maintained, for example, the horizontal funnel or lampshade setup.

\section{Interrogation of representation}

Each representation carries varying levels of disciplinary and pedagogical affordances. Those with technical terminology possess a higher level of disciplinary affordance; for example, Fig. 17. There are similarities between Figs. 14-16 with regards to the nature of the Big Bang. All of them convey the "point in space" alternative conception. This is perhaps another instance where representations need captions to highlight these appresented alternative conceptions. This appresented information can only be discerned with the appropriate level of disciplinary knowledge, and/or guidance by the teacher or educator. Figures 17 and 18 move away from this "point in space" representation, and focus on the concept of inflation, which itself is a dense packing of disciplinary ideas. Some of these representations also aim to reveal the "nature" of the various epochs by using "artistic impressions," actual data, or simulations.

In Fig. 16, we see a focus on the WMAP satellite, as this representation is created to convey the science enabled by WMAP. An expert with a high level of disciplinary discernment would mentally transduct the scale of the WMAP satellite compared with the galaxies. A novice without disciplinary knowledge of scales would need scaffolding by the teacher in order to explain the representations of relative scales. The same applies to Big Bang "point in space" representation.
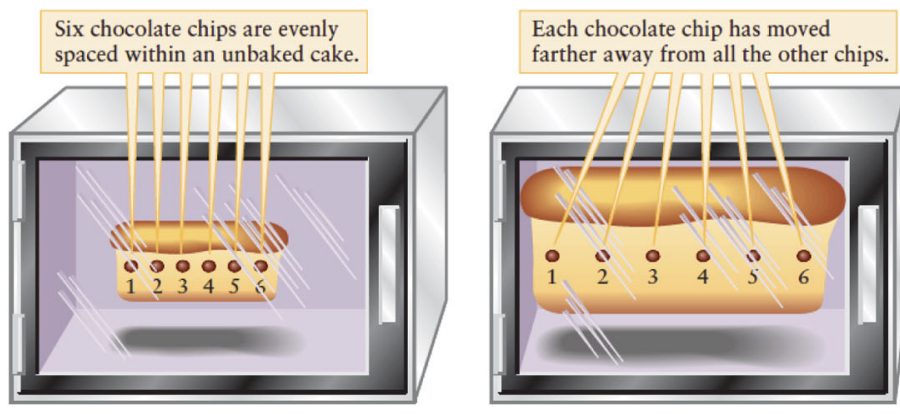

\section{Figure 26-2}

The Expanding Chocolate Chip Cake Analogy The expanding universe can be compared to what happens inside a chocolate chip cake as the cake expands during baking. All of the chocolate chips in the cake recede from one another as the cake expands, just as all the galaxies recede from one another as the universe expands.

FIG. 19. The expanding Universe representational analogy using chocolate chip cake. Freedman, Geller and Kaufmann [101]. Used with permission. 


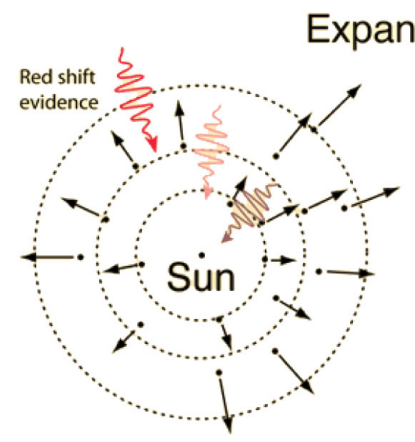

$\mathrm{H}=71 \mathrm{~km} / \mathrm{s} / \mathrm{Mpc}$

FIG. 20. The expanding Universe representational analogy combining two representations, in the form of a translational move supporting a shift in meaning from spatial links with redshift to raisin bread analogy. Image credit: Georgia State University.

Universe, and the raisins are the clusters of galaxies. This is often developed into a visual representation and has had many variations as shown in Figs. 19-21.

Another instance for example is Fig. 20, where the combination of the two representations involve linking of

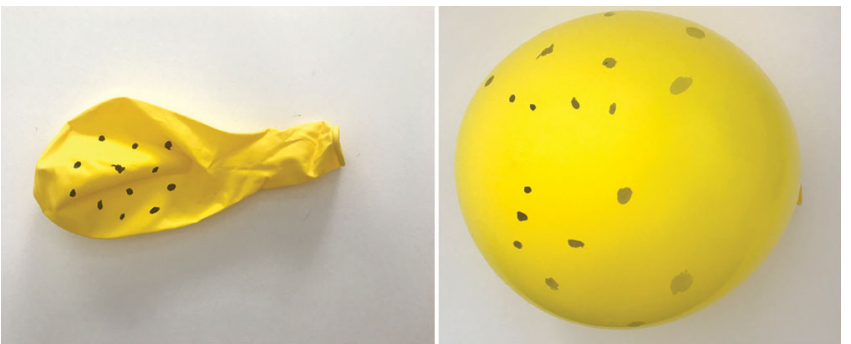

FIG. 23. Expanding balloon with painted dots. Notice how inflating the balloon causes not only "space" to expand, rather also the "galaxies-cluster of galaxies" to expand. An example where teacher scaffolding is necessary to clarify analogies. Image credit: Saeed Salimpour.

redshift representation (left) to the spatial expansion of the bread (cosmological expansion of space).

Another variation of the expansion of space analogy is that of a rubber band being stretched. In this case the concept concerns how the expansion of space causes the wavelength of light to be stretched to longer wavelengthscosmological redshift-shown in Fig. 22. The key idea here is to differentiate cosmological redshift from doppler
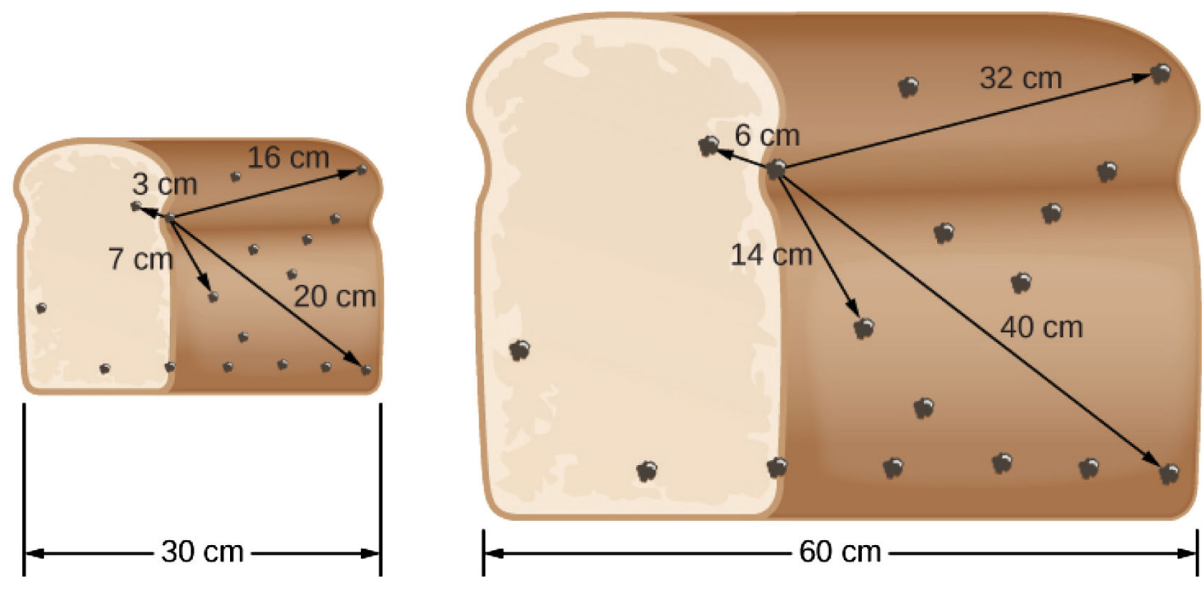

Figure 26.17 Expanding Raisin Bread. As the raisin bread rises, the raisins "see" other raisins moving away. More distant raisins move away faster in a uniformly expanding bread.

FIG. 21. Another variation of the representational analogy showing an expanding Universe. Image credit: Fraknoi et al. [102].

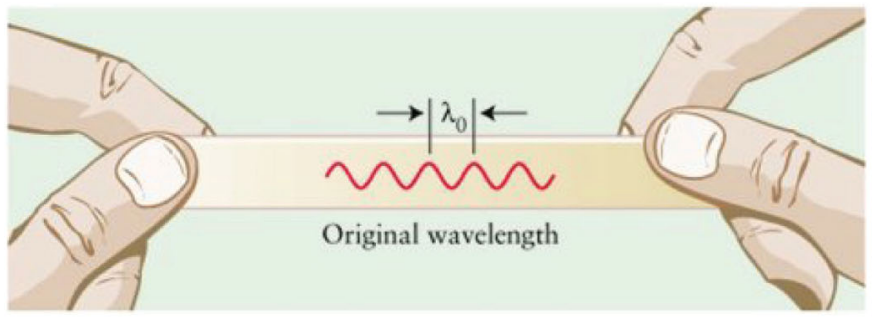

(a) A wave drawn on a rubber band ...

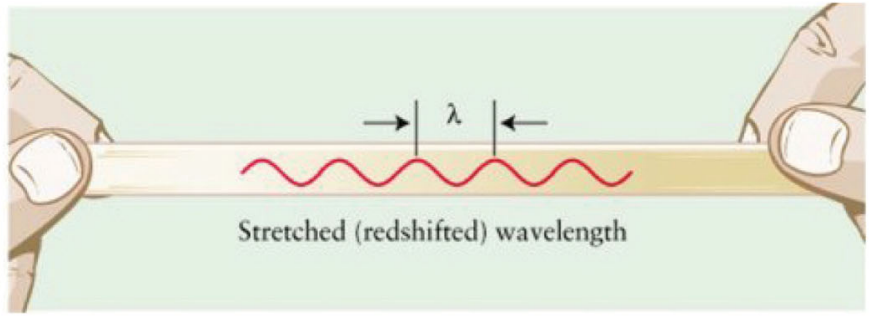

(b) ... increases in wavelength as the rubber band is stretched.

FIG. 22. Cosmological redshift, the expansion of space causing the wavelength of electromagnetic radiation to be stretched. Image credit: Freedman, Geller and Kaufmann [101]. Used with permission. 

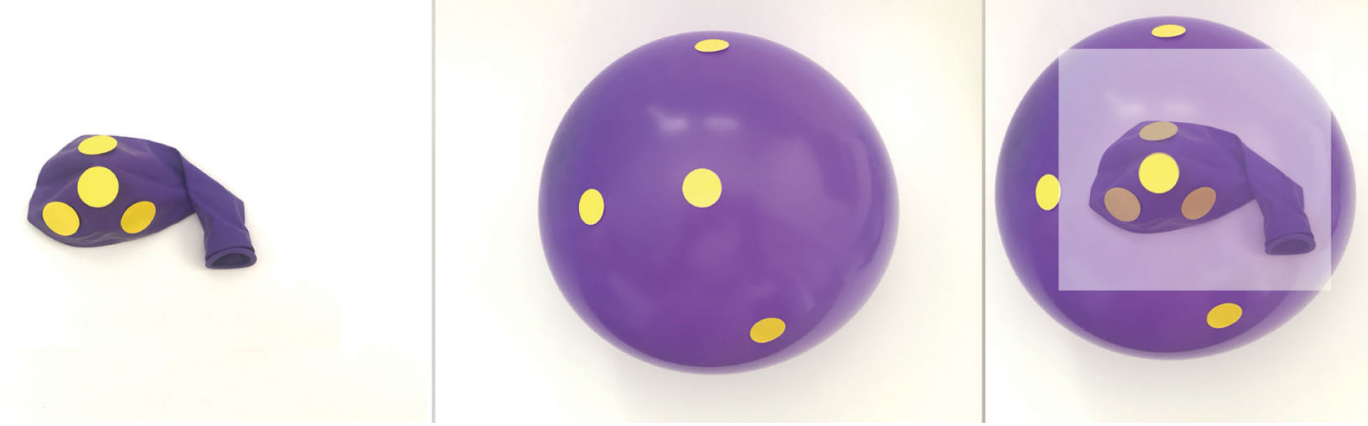

FIG. 24. Expanding balloon with rigid dots. Notice that in this physical demonstration of the analogy, the concept is slightly better communicated. The galaxies themselves are not expanding. Teacher scaffolding is still required to make sense of a potential question that will often arise "What is the Universe expanding into?". Image credit: Saeed Salimpour.

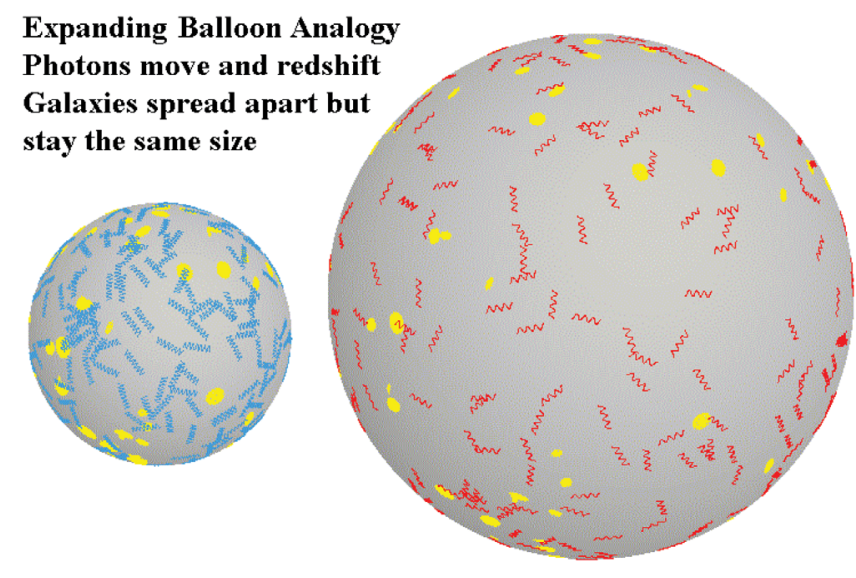

FIG. 25. Expanding balloon analogy showing the stretching of wavelength due to cosmological redshift. Image credit: http:// www.astro.ucla.edu/ wright/balloon0.html.

redshift, the latter being the result of relative motion, whilst the former is due to space itself expanding.

The expanding balloon analogy was proposed in the 1930s [103] as a representation for an expanding Universe.

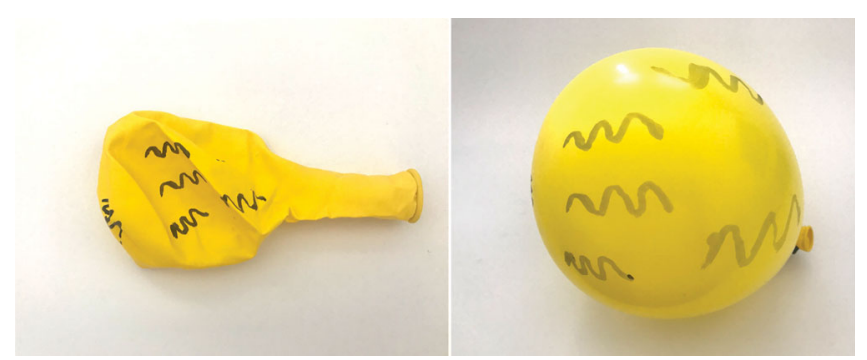

FIG. 26. Expanding balloon wavelength analogy. Here the aim is to communicate the concept that the wavelength of light stretches as the result of space expanding-cosmological redshift. Keen observers would highlight that the wavelength is not actually getting longer, rather the entire waveform is getting larger. Once again, the limit of analogies requires teacher scaffolding. Image credit: Saeed Salimpour.
However, this representation is limited to conveying a small section of reality. The analogy begins by asking the student to imagine a deflated balloon (representing the Universe or space) that has dots (representing galaxies) drawn on it. As the balloon is inflated the space between the dots increases, hence representing the expansion of space. If this analogy is physically demonstrated students will soon realize that the dots are also expanding as shown in Fig. 23. This gives the impression that galaxies are themselves expanding. The interrogation of representations when considering such simplified analogies could include characterizing the structural alignment between elements. This can to a certain degree be encapsulated within appresentation. Structure mapping [50] allows us to identify how the structural relationship in the analogy breaks down when using painted dots. Considering the balloon-painted dots versus Universegalaxies, in the latter the galaxies are not expanding.

Over the years, the dots have been replaced by rigid sticky dots, as shown in Fig. 24, which ensures the galaxies are not expanding. However, the analogy and representation raise some lingering conceptual issues, the most notable: "What is the balloon (Universe) expanding into?" This representation has evolved and now regularly uses a wavelength of light to depict how the stretching (expansion) of space causes the wavelength of light to be stretched to longer wavelengths as shown in Figs. 25 and 26. This is a variation on the rubber band version shown in Fig. 22. Using the AOR framework we can analyze these above analog representations. For the purpose of this study, we will consider the example of the rubber band representation (Fig. 22) as presented in Table V.

\section{TRANSLATION OF DISCIPLINARY REPRESENTATIONS}

This section is aimed at tracing the pathway of representations from disciplinary to pedagogical. Through the analysis, some key aspects of this pathway were identified. First, at sophisticated target audience levels the representations come more in the form of formal graphs 
TABLE V. Unpacking the representation of the rubber band analogy for cosmological expansion.

1. Dimension of representation

The mode of this representation is a static image referencing an embodied material experience, but incorporates the symbol lambda $(\lambda)$, which is a disciplinary semiotic resource to denote wavelength. The representation, however, is purely pedagogical. It should be emphasized that even pedagogical representations will no doubt incorporate disciplinary semiotic resources, as they are trying to teach the disciplinary norms. The art aesthetic in terms of design principles or more specifically the visual grammar employed uses the color red to draw attention to the wavelength of light.

\section{Features of representation}

This representation uses a key mathematical notation lambda $(\lambda)$. The caption in the image does not explain the significance of lambda. However, by using the two arrows and vertical lines, the representation shows that lambda represents wavelength (distance between successive peaks).

\section{Translation of representation}

This representation when combined with the representation of the expanding balloon shows the wavelength of light gets longer (stretched). This effect is known as redshift and is a result of cosmological expansion (Fig. 21).

\section{Interrogation of representation}

The representation does require a certain degree of disciplinary discernment with regards to $\lambda$ and $\lambda_{0}$. The presence of disciplinary affordances in representational analogies are perhaps central to their work as bridges; for example, the link between the expansion of space and wavelength increase.

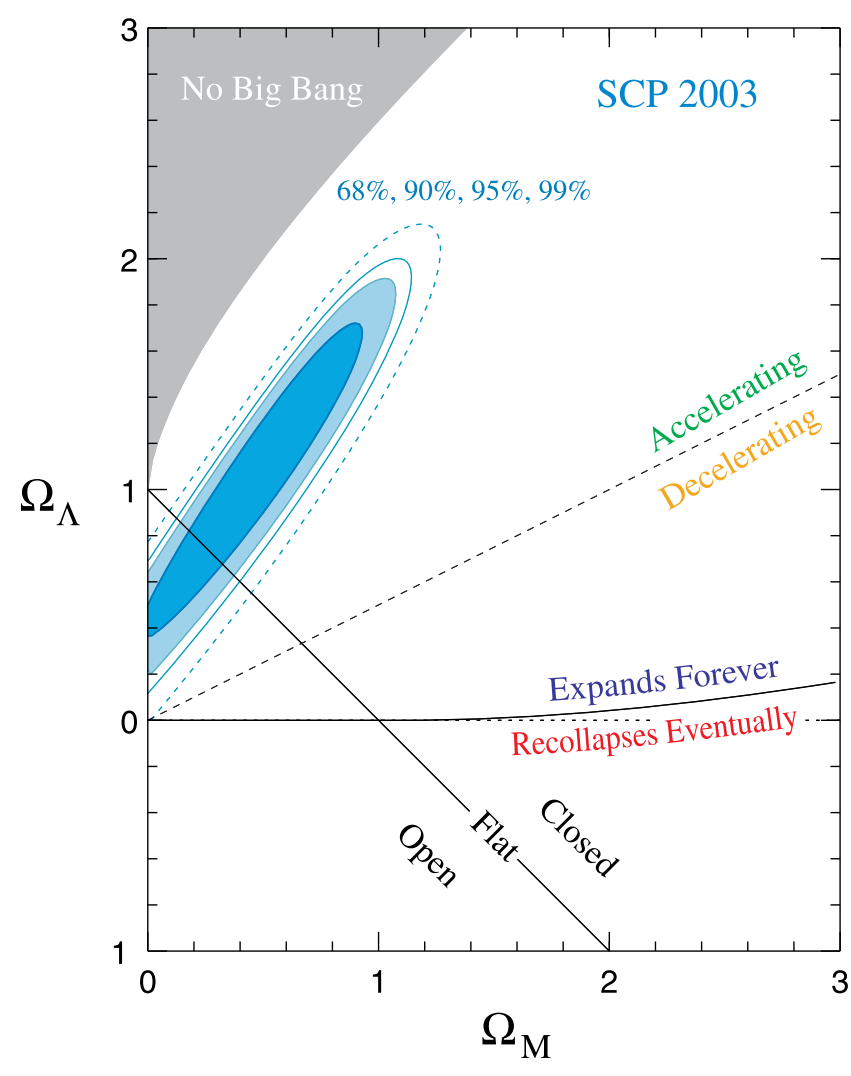

FIG. 27. Original figure from research group, showing the various scenarios relating to the relationship between the matter density $\left(\Omega_{M}\right)$, and energy density $\left(\Omega_{\Lambda}\right)$. Image credit: Knop et al. [104]. or plots using a range of disciplinary semiotic resources often seen in peer-reviewed scientific journal publications (e.g., Figs. 27, 31). These representations are often simplified when used in lower levels, or they may have extra information in the form of annotations added to them to explain different parts of the representation. This is encompassed by the notion of translation, an example of which is seen in Fig. 27. The representation, by the addition of annotations that explain key parts, has evolved from the original published in a peer-reviewed journal, to the one shown in Fig. 28, used in an introductory astronomy textbook. Figure 29 shows a version used for public outreach where the symbolism associated with disciplinary knowledge is removed entirely, although the format of a scientific graph is maintained. An expert looking at Fig. 27, given their disciplinary fluency, would be able to mentally transduct into Fig. 28. For an expert, the disciplinary aesthetic associated with Fig. 27 involves the clarity in representation of the implications of different models.

Sometimes a particular representation at higher levels is broken up into focused concepts. This is demonstrated by Fig. 30, which shows how the representation of the evolutionary timeline of the Universe is broken up into multiple representations each focusing on different concepts. The concepts include how the temperature of the Universe has changed, the use of redshift, the frequency of emissions, and fluctuations in the $21 \mathrm{~cm}$ hydrogen emissions. The focus of this representation is on the Dark Ages followed by the Epoch of Reionization-when the Universe went from being a cold dark place, to the first 


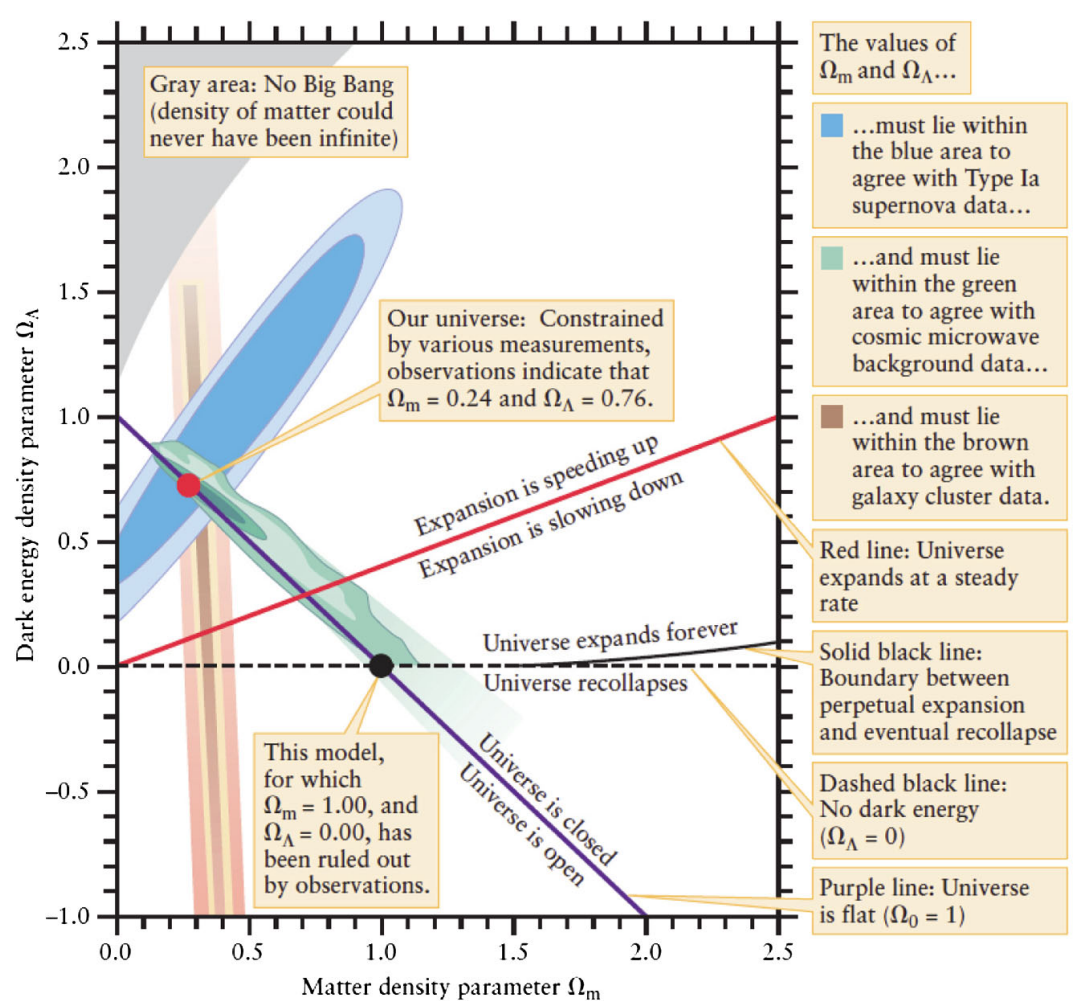

Figure 26-19

Limits on the Nature of the Universe The three regions on this graph show values of the mass density parameter $\boldsymbol{\Omega}_{\mathrm{m}}$ and the dark energy density parameter $\Omega_{\Lambda}$ that are consistent with various types of observations. Galaxy cluster measurements (in brown) set limits on $\Omega_{\mathrm{m}}$. Observations of the cosmic microwave background (in green) set limits on the sum of $\Omega_{\mathrm{m}}$ and $\Omega_{\mathrm{A}}$ : A larger value of $\Omega_{\mathrm{m}}$ (to the right in the graph) implies a smaller value of $\Omega_{\Lambda}$ (downward in the graph) to keep the sum the same, which is why this band slopes downward. Obsenvations of Type Ia supernovae (in blue) set limits on the difference between $\boldsymbol{\Omega}_{\mathrm{m}}$ and $\Omega_{A}$; this band slopes upward since a larger value of $\Omega_{\mathrm{m}}$ implies a larger value of $\Omega_{\Lambda}$ to keep the difference the same. The best agreement to all these observations is where all three regions overlap (the red dot). (The Supernova Cosmology Project/R. A. Knop et al.)

FIG. 28. Modified figure used in an introductory astronomy textbook. Image credit: Freedman, Geller, and Kaufmann [101]. Used with permission.

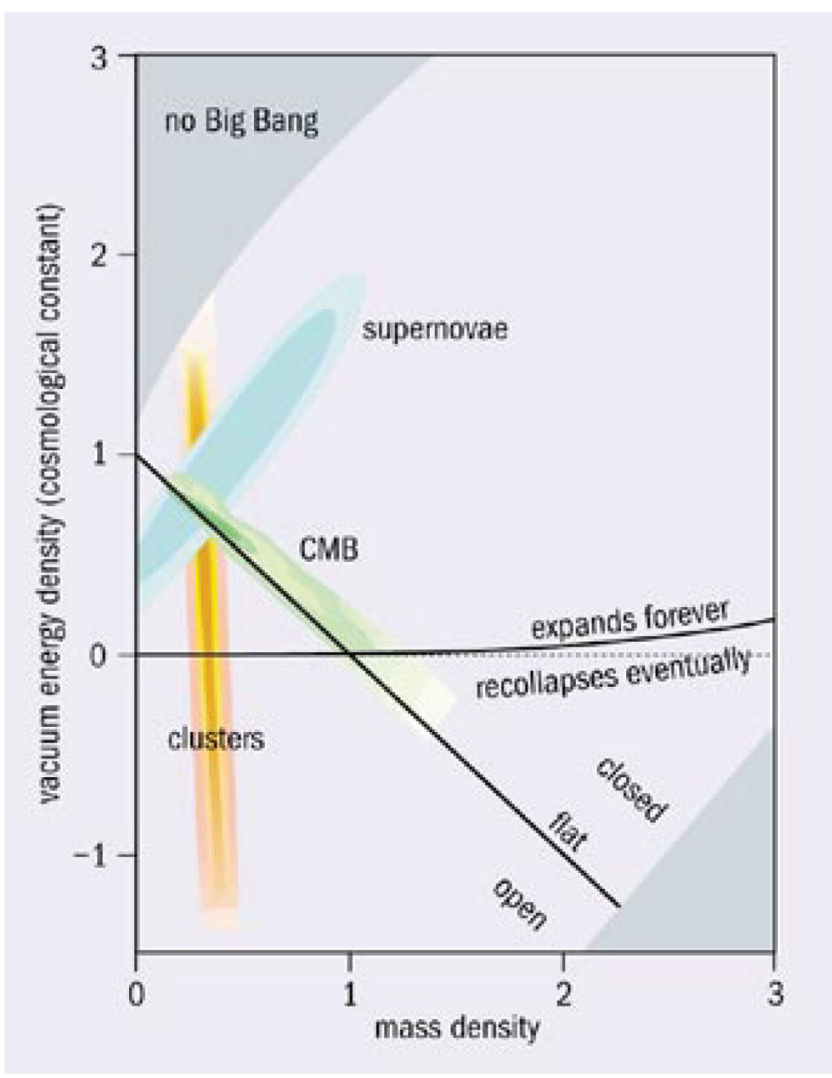

FIG. 29. Modified figure used in a media release. Image credit: https://cerncourier.com/a/on-the-trail-of-dark-energy/. stars or galaxies forming, and "heating or lighting" up the Universe [105,106]. The representation is aimed at professionals, and members of the public with a higher degree of disciplinary knowledge. This figure has its origins in a peer-reviewed journal (Fig. 31), and as such has undergone a translation from the disciplinary context in the form of a simplification to be presented as Fig. 30. This translated representation is aimed at communicating key ideas to a more general but informed audience. Comparing the representation in Fig. 30 with those of Figs. 14-18 shows how some of the key elements such as time and temperature are maintained.

As noted earlier, the translation of a representation can involve two or more different representations being combined to create another representation-combination. This is illustrated in Fig. 32, where the representation showing the geometry of the Universe (Fig. 13) is combined with a data visualization of the $\mathrm{CMB}$ from BOOMERANG (top panel), including three simulations of the CMB for the different geometries of the Universe. The strategic interplay between models of the curvature of space and observational data is another example in this instance of disciplinary aesthetics.

These variations of the same representation require choices to be made by the creator of the representation based on its pedagogical or disciplinary aim. These decisions in the case of combination include what information is maintained from each disciplinary representation, 
(a)
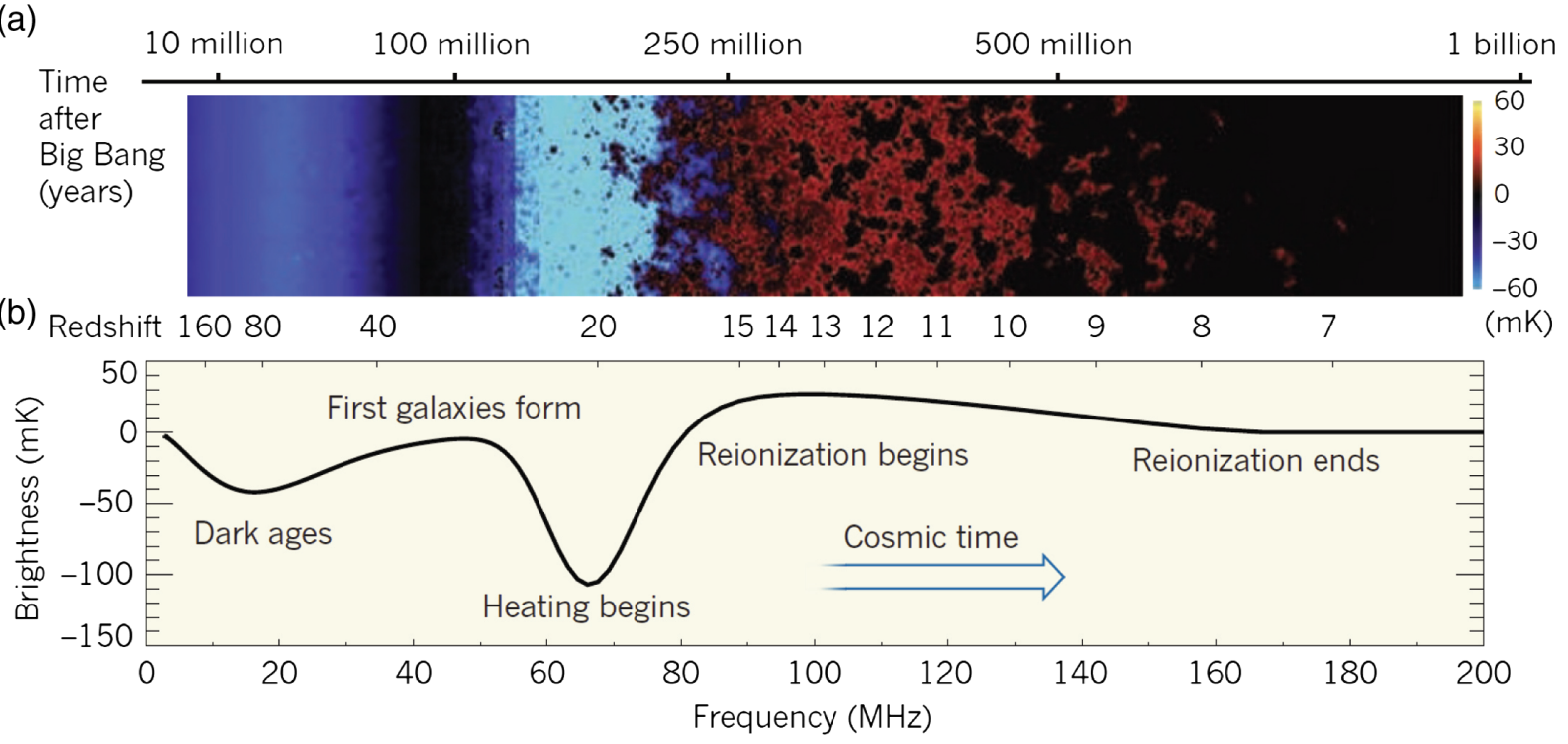

FIG. 30. The evolutionary timeline of the Universe. Image credit: Pritchard and Loeb [106].

or in the case of simplification, what disciplinary information is made explicit, appresented or removed entirely.

Often discussions about cosmology and the Universe involve showing the classical image of the CMB (Figs. 1 and 33). Looking at Fig. 1, to a novice with limited disciplinary knowledge, it would be challenging to understand the conceptual information contained within the image. The novice would in most cases appreciate the everyday and art-aesthetic associated with the image, which possesses a certain mystery and beauty. This would lead them to question: What do the colors mean? Why is the image oval shaped? What does the pattern mean? High school textbooks may use the caption that states the image is of the CMB, the glow from the Big Bang. The caption that goes with the original image release by European Space Agency (ESA) states (Fig. 1): "The anisotropies of the Cosmic Microwave Background (CMB) as observed by Planck. The CMB is a snapshot of the oldest light in our Universe, imprinted on the sky when the Universe was just 380000 years old. It shows tiny temperature fluctuations that correspond to regions of slightly different densities, representing the seeds of all future structure: the stars and galaxies of today." Apart from the complexity of information contained within the image, the shape of the image itself presents challenges. Map projections play an important role in cosmology when all-sky measurements are mathematically processed to become a $2 \mathrm{D}$ flat image. Students need the appropriate scaffolding to appreciate why such projections are useful.

To gain insights from the image, scientists need to extract information, for example plotting the power spectrum of the CMB, as is seen in Fig. 34. Reading the representation of the power spectrum and connecting it to the all-sky CMB image requires a high level of disciplinary discernment.
Scientists with disciplinary fluency will mentally transduct to create connections between the representations of CMB and its power spectrum. Introductory textbooks will translate the representation to increase the pedagogical affordance (Fig. 35). In Fig. 35 the graph is simplified to include only two variables, annotations are used to explain key features of the plot, and a caption that gives more information. Furthermore, looking at the representation in a popular science article (Fig. 36), there is evidence of how the power spectrum (Fig. 34) and the CMB image (Fig. 1) are combined and simplified to increase the pedagogical affordances. The use of an explanatory caption in Fig. 35 increases the pedagogical affordance by deemphasizing the detail in the graphical axes and inserts the best fit story. The addition of a visual interpretation of what this might mean in terms of patterns of spatial distribution contributes to increasing the pedagogical affordance.

\section{DISCUSSION}

The research questions posed in this study are answered based on the analysis in the previous section. In answering the first research question: "What does the landscape of cosmology representations look like for different audiences and different conceptual dimensions?" this study reveals that the landscape of cosmology representations is comprised of key visualizations that can be categorized into four main themes: size and scale, spacetime location, composition, and evolution. Overall, there are more representations in the theme for evolution, perhaps owing to progress in the field with experimental successes, or because they bring together curiosity piquing concepts that are unresolved and areas of current research. There is a lack of representations aimed specifically at the HS level. 

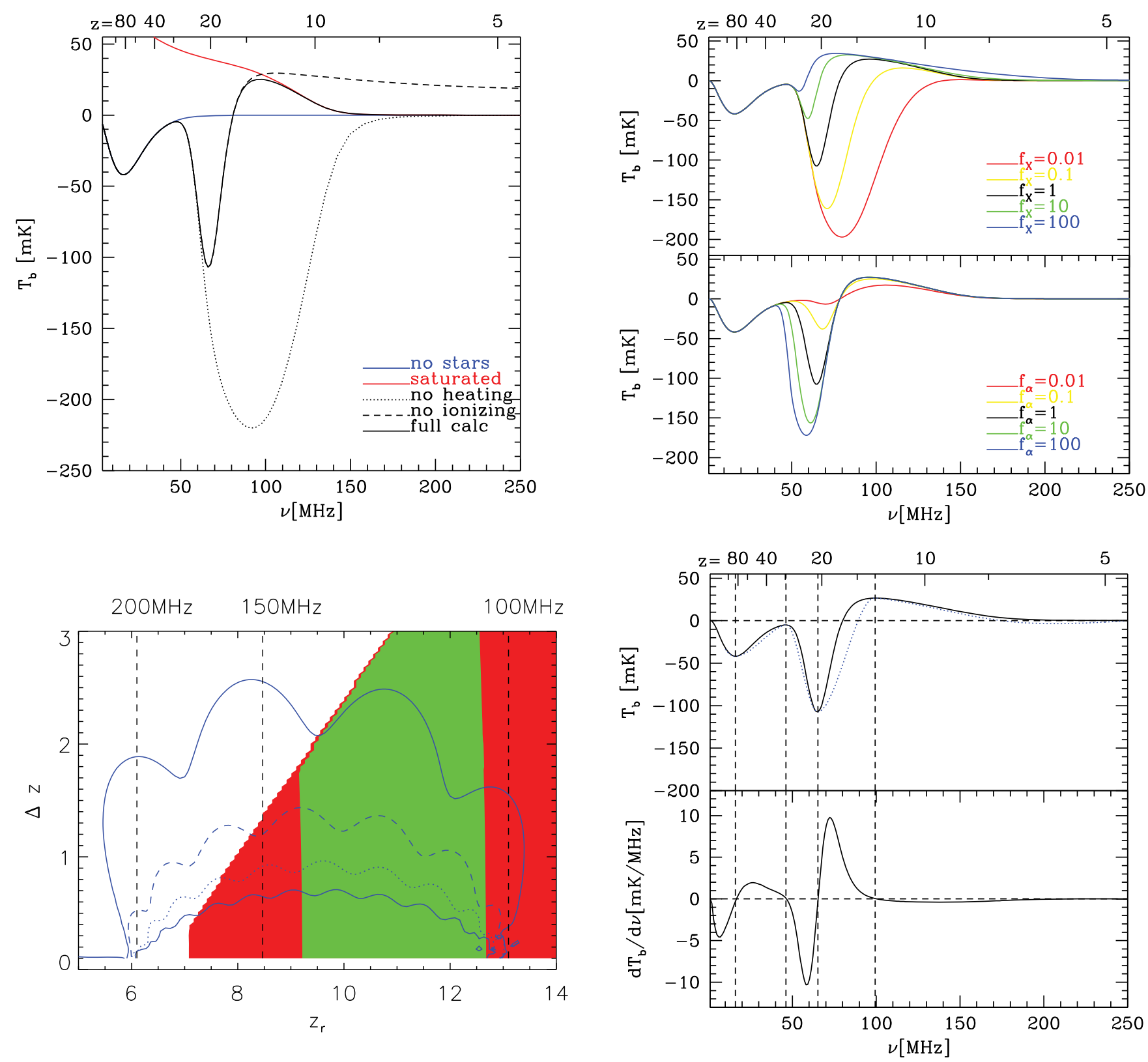

FIG. 31. Representations from peer-reviewed journal article. Image credit: Pritchard and Loeb [105].

Most representations used at either PO or UG levels tend to be used also at HS level, sometimes with modifications. The representations used in PO vary widely in relative disciplinary and pedagogical affordances. Some are simplified enough to be used in high school, but even these may not always have the necessary supports and unpacking around which to build a conceptual progression and student understanding.

In answering the second research question: "How can we systematically describe the varying nature and purpose of representations in cosmology education?" this study found that the representations created at various levels (PO, HS, UG, PG, and PRO) each offer a different balance between pedagogical and disciplinary affordances. Representations at the levels of PRO and PG will make use of semiotic resources that are unique to the discipline, for instance, the use of very specific mathematical notations or disciplinespecific terminology. In the context of PRO and PG, there is some element of pedagogy; however, many of the concepts are appresented; cf. Ref. [97]. At the UG level, the pedagogical affordances of the representations are higher. There is an emphasis on the building of representational awareness, which is characteristic of sophisticated disciplinary knowledge or discernment; cf. Ref. [64]. UG students are assumed to have some of the disciplinary semiotic resources in their knowledge base. At the HS level students are new to the discipline and therefore require scaffolding to allow them to the learn the disciplinary 


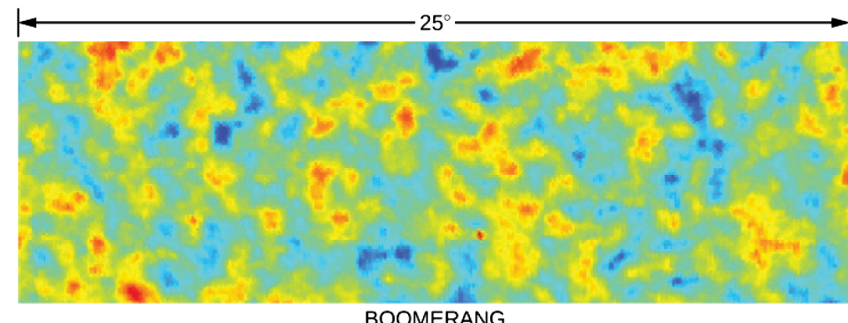

BOOMERANG
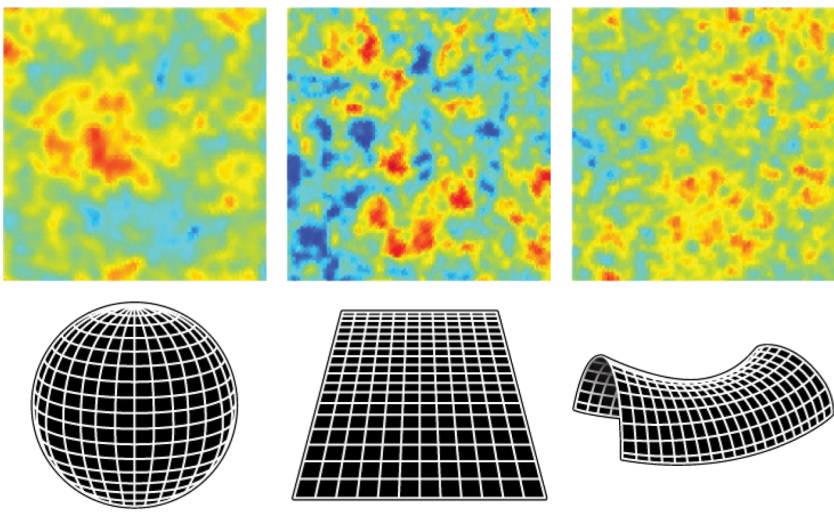

FIG. 32. An example of combination, using data generated representation, together with computer modeling, and the possible geometries, seen in Fig. 13. Image credit: OpenStax Astronomy Textbook modification of work by NASA [102].

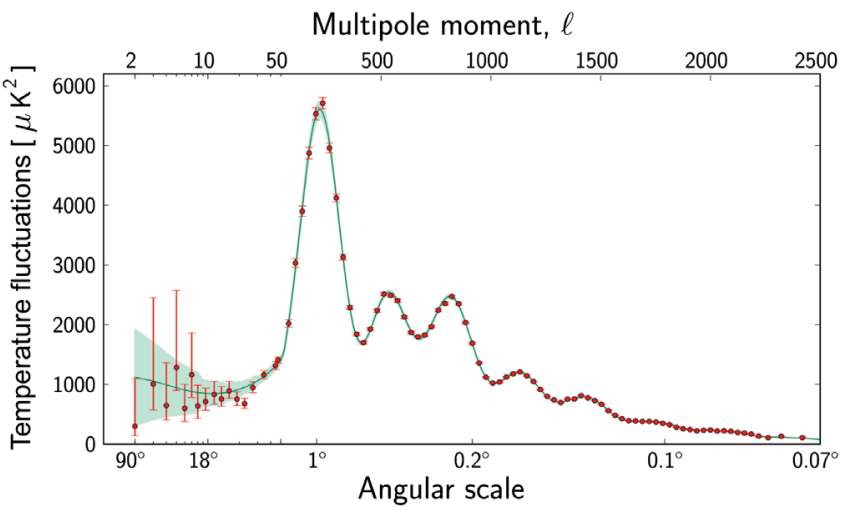

FIG. 34. Power spectrum of temperature fluctuations in the cosmic microwave backgrounds. Image credit: ESA and the Planck Collaboration.

knowledge assumed in and underpinning the representations. Representations at this level need to have a high level of scaffolding, which includes interpretive comments to ensure they communicate the underlying concepts effectively to students. This is a vital characteristic that representations with a high degree of pedagogical affordance should possess. The use of annotations and captions are powerful bridges that help students use everyday experience to build representational meaning around

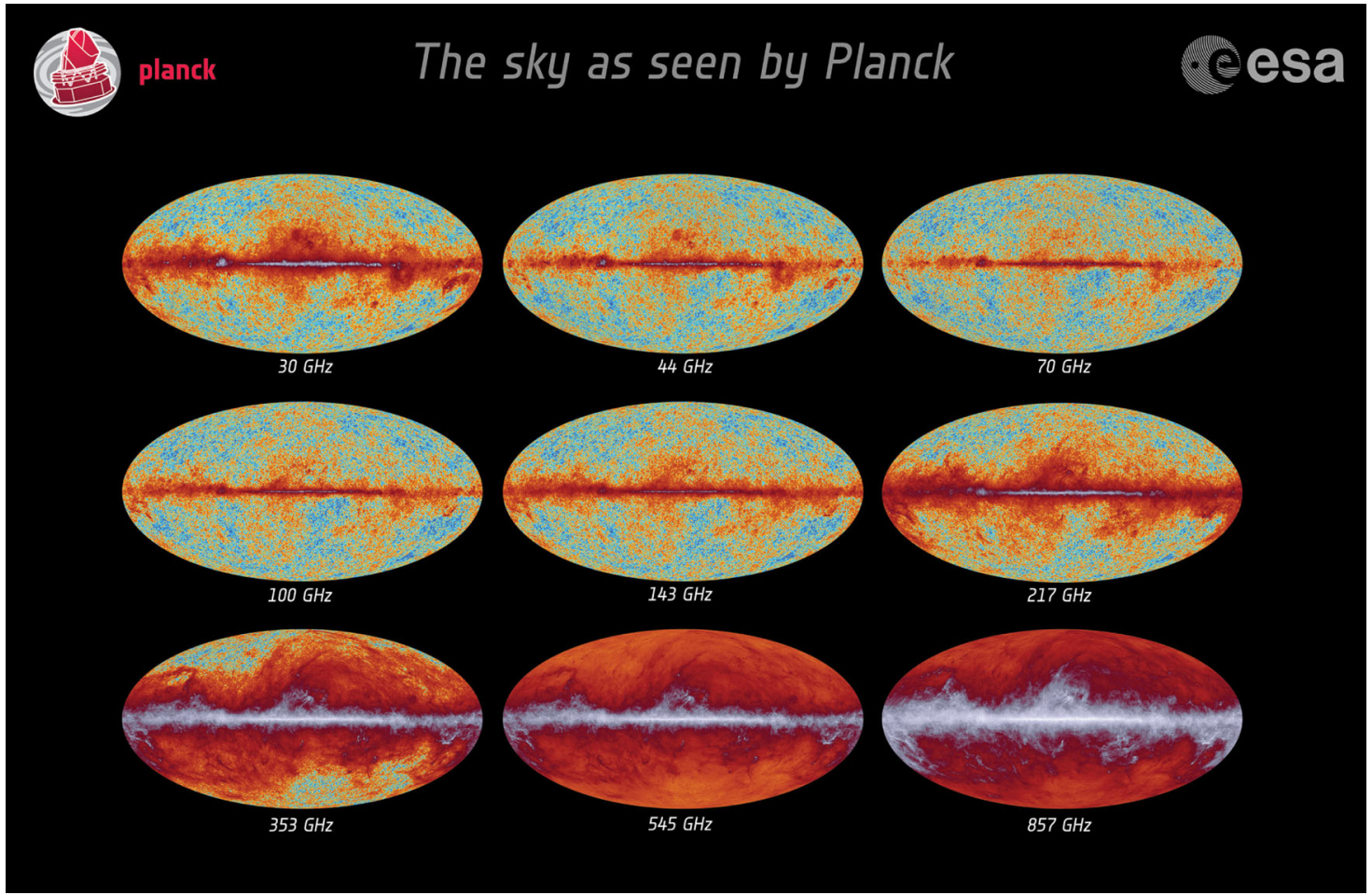

FIG. 33. Measurements of the cosmic microwave background in various frequencies. The data underlying these images is used to reconstruct the signal from the cosmic microwave background, which is masked by emissions from various phenomena and dust. Image credit: ESA and the Planck Collaboration. 


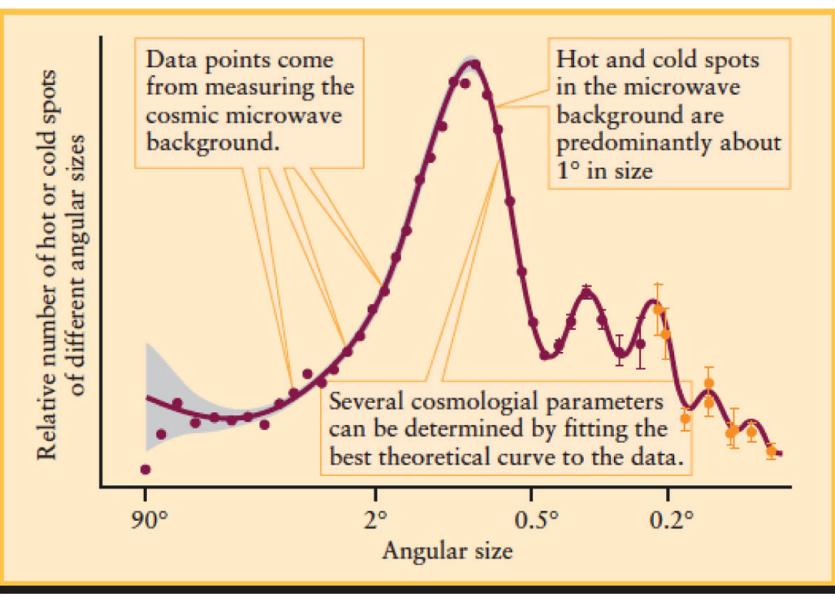

Figure 26-21

Sound Waves in the Early Universe Observations of the cosmic background radiation show that hot and cold spots of certain angular sizes are more common than others. A model that describes these observations helps to constrain the values of important cosmological parameters. Most of the data shown here is from the Wilkinson Microwave Anisotropy Probe; the data for the smallest angles (at the right of the graph) come from the CBI detector in the Chilean Andes and the ACBAR detector at the South Pole. (NASA/WMAP Science Team)

FIG. 35. Power spectrum of the cosmic microwave background, as seen in an introductory astronomy textbook. Image credit: Freedman, Geller, and Kaufmann [101]. Used with permission.

sophisticated disciplinary concepts and can make appresented information become more explicit.

In answering the final research question: "How is the translation made between disciplinary representations and representations in cosmology education?" this study, in approaching and unpacking these semiotic resources, found that there is a need for teachers to be aware of the various elements of these representations. This allows teachers to help students unpack representations, interpret analogies, and anticipate problems with appresentation and with complexity in the compound images. The development of the AOR framework as part of this study can serve as a valuable tool in this process of translating disciplinary representations to pedagogical representations.

Using the work of Airey and Eriksson [108], each of the levels (PO, HS, UG, PG, and PRO) can be situated in the disciplinary-pedagogical space, as shown in Fig. 37. The translation from disciplinary to pedagogical is a complex process and depends on the information or concepts that need to be communicated. It is obvious that representations created for a higher level are not always suitable at lower levels. They may not carry the same level of pedagogical affordances and as such need to be translated via simplification, combination, or analogies to provide pedagogical affordance. Furthermore, if a disciplinary representation is simplified to be used in a pedagogical context, then care must be taken to ensure the knowledge the representation aims to teach is maintained. Representations created to be used in a pedagogical context need to be designed to make the concept readable by the student. This can involve making visible or explicit the disciplinary concepts that are often appresented.

The target audience in the PO category is broad and so the levels of knowledge vary. The intention for a representation in the PO category is to communicate a concept and often the associated aesthetic. In particular these often communicate a sense of awe and wonder attached to the sheer spatial and temporal magnitudes, and the beauty and complexity of the images. Representations for PO range from those that keep disciplinary affordances to a minimum, to those that have a high level of disciplinary affordance. Often the level of pedagogical affordance in PO representations is maintained at a medium level.

The AOR framework can provide a guide for teachers and educators on various levels. It provides scaffolding to orient students to how to read representations or to unpack representations; whether they are canonical, or student generated. In following the work of Airey and Eriksson [108], the process of "unpacking" a semiotic resource (representation) that has a high disciplinary affordance so that it is accessible to the student in essence involves increasing the pedagogical affordance as shown in Fig. 38. Although there are representations that can have both high disciplinary and pedagogical affordances (light green; top right quadrant), in order to teach students the disciplinary norms the representation needs to be "unpacked" to reduce the density of "disciplinary shorthand-an accepted way of sharing knowledge with other experts within the discipline" [108] (p. 100). It could be argued that the unpacking arrow could move horizontally, maintaining the level of disciplinary affordance, whilst increasing the level of pedagogical affordance. This could be true for high-level representations used by professionals, where they aim to educate their peers in the new research.

The translation from a representation with high disciplinary affordance to create a representation with high pedagogical affordances can occur in a number of ways that are described in the AOR framework. Thus, the AOR framework becomes a useful tool for teachers to become aware of and help unpack what is at stake in clearly representing disciplinary concepts in a way that can be understood by students. This may help in unpacking or evaluating textbook representations and also devising representation construction tasks where students generate, evaluate and refine representations that help them in their disciplinary discernment of canonical representations.

\section{IMPLICATIONS FOR EDUCATION}

Representations are discursive tools through which we understand and communicate concepts that can be intangible and counterintuitive. As such, representations 


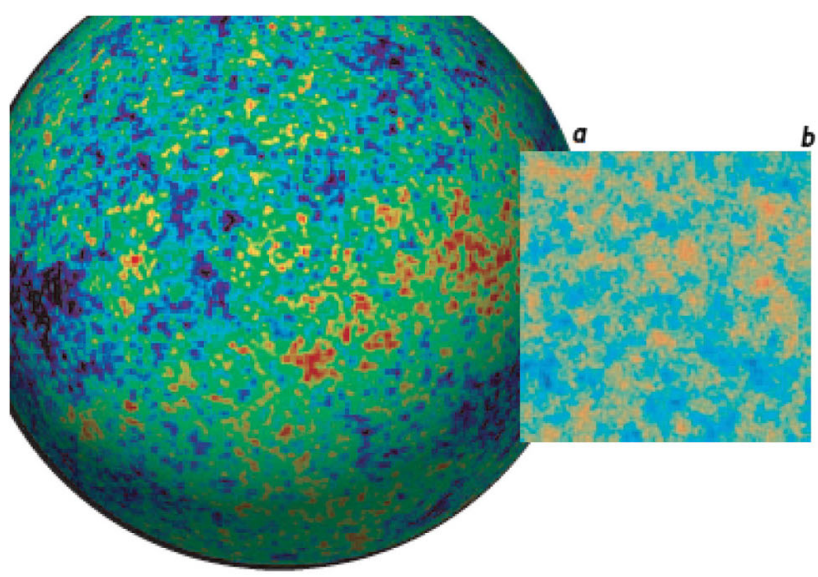

OBSERVATIONS OF THE CMB provide a map of temperature variations across the whole sky $[a]$. When researchers analyze portions of that map $[b]$, they use band filters to show how the temperature of the radiation varies at different scales. The variations are barely noticeable at large scales corresponding to regions that stretch about 30 degrees across the sky $[c]$ and at small scales corresponding to regions about a tenth of a degree across [ $e$ ]. But the temperature differences are quite distinct for regions about one degree across [ $d$ ]. This first peak in the power spectrum [graph ot bottom] reveals the compressions and rarefactions caused by the fundamental wave of the early universe; the subsequent peaks show the effects of the overtones.

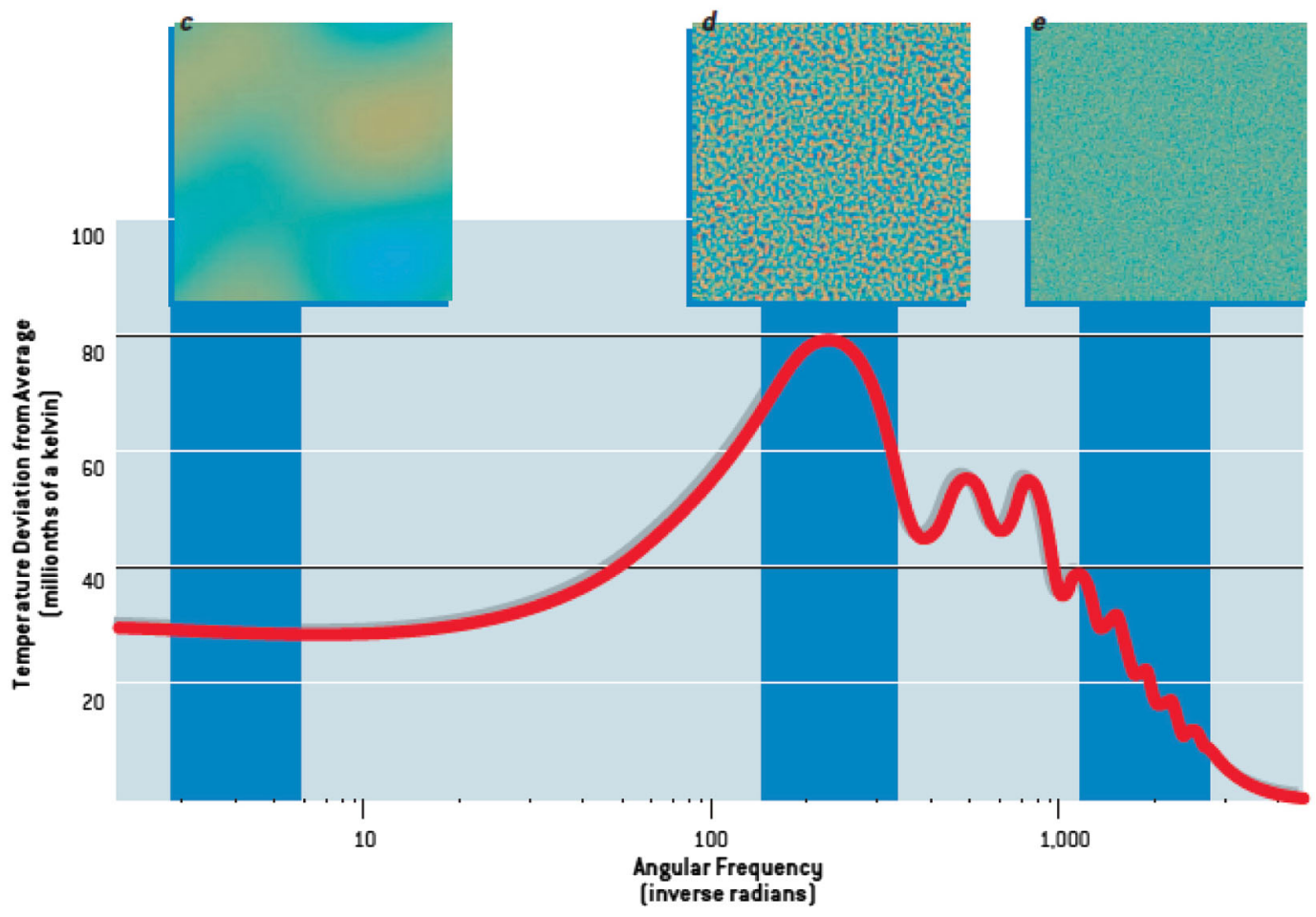

FIG. 36. Power spectrum of the cosmic microwave background, as seen in a popular science article. Image credit: Hu and White [107].

encompass a complex interconnected web of meaningmaking and students require support in navigating this web. If students are to successfully comprehend the meaning(s) encompassed in representations then teachers need to be able to identify the purpose, structure and features of the representations. The AOR framework provides teachers and students with a metalanguage for critically analyzing and supporting the development of disciplinary discernment needed to unpack representations. The framework and its associated metalanguage provide supports through which teachers and students can better understand and critique representations. It could be argued that teachers without the disciplinary background would find working with the AOR challenging. However, the AOR itself is a tool for gradually developing disciplinary knowledge, with appropriate support.
Furthermore, in order to use representations flexibly to problem solve, speculate, and explain, students need to be able to generate their own versions of these canonical representations. To do this, it is helpful for students to understand the histories, complexities, and limitations of the representations they encounter. These sets of skills are part of representational competence, which has been defined as the collection of skills that "involve interpreting, generating, and manipulating external representations to support learning, problem solving, and communicating in STEM fields" [109] (p. 205). However, the term metarepresentational competence [47,110] is also used to emphasize explicit knowledge about the nature and function of representational structure, and their affordances. These terms overlap in literature. Metarepresentational competence is described as 


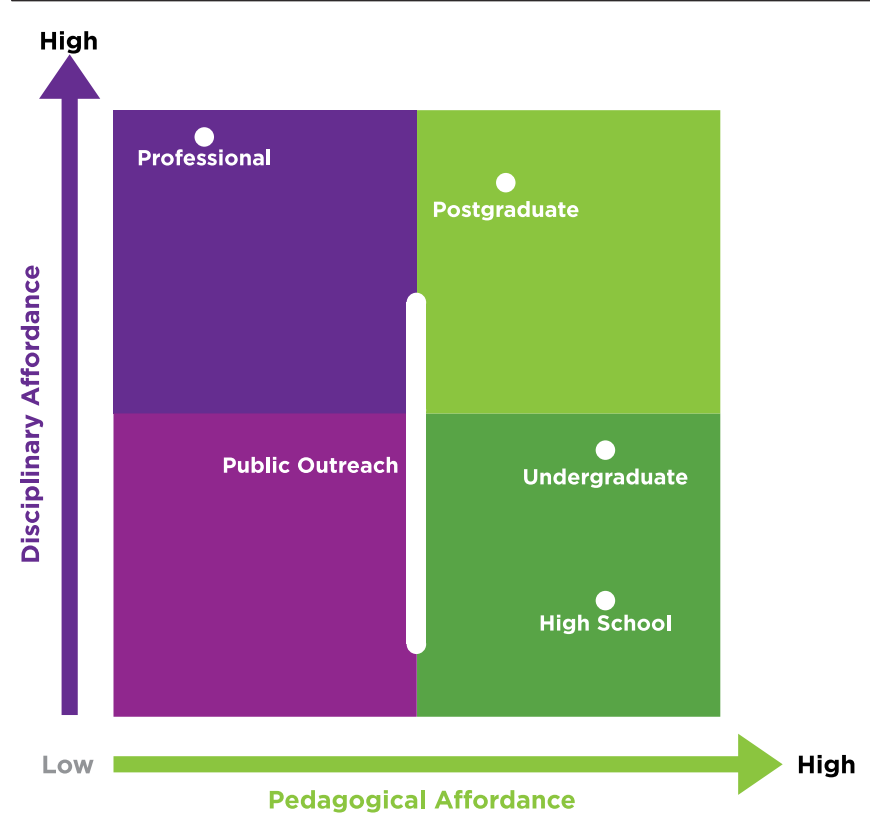

FIG. 37. The distribution of levels based on the disciplinarypedagogical space. Adapted from the work of Airey and Eriksson [108].

"purviews that transcend the mere production and use of representations" [110] (p. 294). The use of the AOR can be seen as a scaffold for helping build teacher and student metarepresentational competence $[47,48]$.

We need to emphasize that the AOR framework itself is not the activity; it is a tool for systematically enabling teachers and students to think critically about using representations in their teaching and learning. An important aspect of students' representational competence is the ability to construct representations to problem solve. A guided

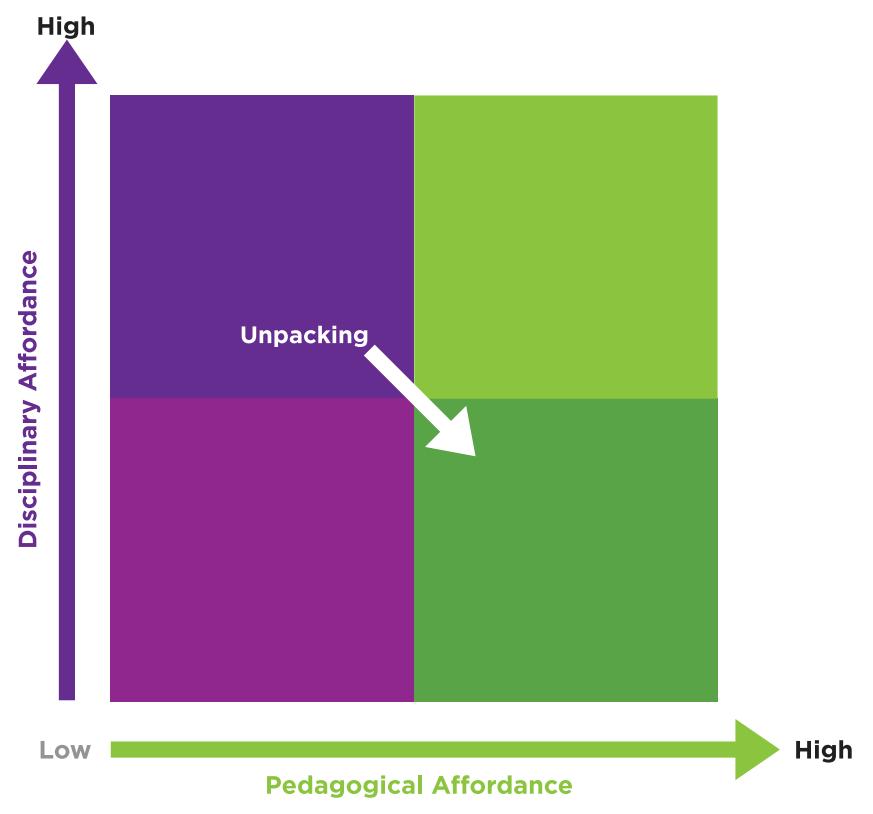

FIG. 38. The idea of unpacking representations. Adapted from the work of Airey and Eriksson [108]. inquiry approach (representation construction) involving students constructing, evaluating and refining representations has been shown to be effective $[17,52,111]$ in supporting learning through teacher scaffolding of representational competence. The manner in which the teacher frames the use of the AOR in their classroom will depend on the context of their unit of study. A teacher new to this pedagogical approach will no doubt require professional development. One approach would be for the teacher to start by creating (or using) a sample representation and going through the AOR framework in their own teaching teams. If the teacher has time, they could involve their students in this pilot analysis. Any new pedagogical approach to teaching and learning requires that the teachers be provided enough support at the beginning. At this stage we are presenting the AOR as tool that has sound theoretical and pedagogical foundations; and has been developed using representations that are currently used in cosmology. A complete teacher's guide with examples is currently in development.

Although the focus of this work has been in the context of classroom teaching and learning, previous studies have highlighted how representations in textbooks can lead to misinterpretation [112,113]. The AOR framework can be useful for authors of educational materials to help guide the development of the representations used in their materials. It will encourage authors to systematically analyze their representations and ensure that they are suitable for their target users. We appreciate the complexity of representing astronomical phenomena on a $2 \mathrm{D}$ surface and this is not a criticism of textbook authors. Being able to identify the key aspects of a representation, the concept that the representation is meant to communicate and how it might be interpreted, could be useful to help avoid alternate conceptions that may unintentionally arise when interpreted by the novice reader.

\section{CONCLUSIONS}

This review is aimed at unpacking current disciplinary and pedagogical representations to highlight how they evolve, and the various disciplinary or pedagogical affordances that support a meaningful reading of these representations. A collection of cosmology representations was compiled into four overarching themes: size and scale, spacetime location, composition of the Universe, and evolution of the Universe, in order to explore the landscape of the most prevalent canonical cosmology representations across a range of media. A qualitative analysis of the representations has allowed the construction of a framework aimed at unpacking representations used in teaching and learning cosmological concepts for their affordances in offering opportunities to develop disciplinary discernment. The anatomy of representations framework is composed of four interacting layers. The outer two layers are a categorization system, while the inner two layers relate to a deeper 
reading and interrogation of the representation. This framework is designed to be a guiding tool and provide a metalanguage for teachers when teaching concepts connected to representations, and also for producers of educational materials. Understanding the features of representations supports the development of the metarepresentational competence students need for interpreting and creating their own representations in cosmology.

\section{ACKNOWLEDGMENTS}

The authors would like to thank the reviewers for their detailed and constructive feedback in preparing this manuscript. Dr. Michael Fitzgerald is the recipient of an Australian Research Council Discovery Early Career Award (Project No. DE180100682) funded by the Australian Government.
[1] U. Eriksson, C. Linder, J. Airey, and A. Redfors, Who needs 3D when the Universe is flat?, Sci. Educ. 98, 412 (2014).

[2] R. Gray, The distinction between experimental and historical sciences as a framework for improving classroom inquiry, Sci. Educ. 98, 327 (2014).

[3] M. Vogelsberger, S. Genel, V. Springel, P. Torrey, D. Sijacki, D. Xu, G. Snyder, D. Nelson, and L. Hernquist, Introducing the Illustris Project: Simulating the coevolution of dark and visible matter in the Universe, Mon. Not. R. Astron. Soc. 444, 1518 (2014).

[4] J. North, Cosmos: An Illustrated History of Astronomy and Cosmology (University of Chicago Press, Chicago, IL, 2008).

[5] R. P. Norris, The Challenge of Astronomical Visualisation, in Astronomical Data Analysis Software and Systems III ASP Conference Series, edited by D. R. Crabtree, R. J. Hanisch, and J. Barnes, Vol. 61 (Astronomical Society of the Pacific, 1994), p. 51, http://adsabs .harvard.edu/abs/1994ASPC...61...51N.

[6] G. R. Kress and T. van Leeuwen, Reading Images: The Grammar of Visual Design, 2nd ed., reprinted (Routledge, London, 2010).

[7] S. Galano, A. Colantonio, S. Leccia, I. Marzoli, E. Puddu, and I. Testa, Developing the use of visual representations to explain basic astronomy phenomena, Phys. Rev. Phys. Educ. Res. 14, 010145 (2018).

[8] V. R. Lee, How different variants of orbit diagrams influence student explanations of the seasons, Sci. Educ. 94, 985 (2010).

[9] B. M. Pena and M.J.G. Quilez, The importance of images in astronomy education, Int. J. Sci. Educ. 23, 1125 (2001).

[10] R. Tytler, Re-imagining science education: Engaging students in science for Australia's future, Review No. 51, Australian Education Review - Australian Council for Educational Research, 2007, https://research.acer .edu.au/aer/3.

[11] V. Prain and R. Tytler, Representing and Learning in Science, in Constructing Representations to Learn in Science (Sense Publishers, Rotterdam, 2013), pp. 1-14.

[12] Visualization in Science Education, edited by J. K. Gilbert (Springer Netherlands, Dordrecht, 2005).
[13] J. K. Gilbert, Visualization: An emergent field of practice, and enquiry in science education, in Visualization: Theory, and Practice in Science Education, edited by J. K. Gilbert, M. Reiner, and M. Nakhleh (Springer Netherlands, Dordrecht, 2008), pp. 3-24.

[14] S. Salimpour, M. T. Fitzgerald, R. Tytler, and U. Eriksson, Educational design framework for a web-based interface to visualise authentic cosmological "big data" in high school, J. Sci. Educ. Technol., https://doi.org/10.1007/ s10956-021-09915-2 (2021).

[15] S. Salimpour et al., The gateway science: A review of astronomy in the OECD school curricula, including China and South Africa, Res. Sci. Educ., https://doi.org/ 10.1007/s11165-020-09922-0 (2020).

[16] S. Salimpour, R. Tytler, M. T. Fitzgerald, and U. Eriksson, Is the Universe infinite? Exploring high student conceptions of cosmology concepts using open-ended surveys (to be published).

[17] Constructing Representations to Learn in Science, edited by R. Tytler, V. Prain, P. Hubber, and B. Waldrip (SensePublishers, Rotterdam, 2013).

[18] R. Tytler, The role of visualisation in science: a response to "science teachers' use of visual representations", Studies Sci. Educ. 57, 129 (2020).

[19] P. J. E. Peebles, Principles of Physical Cosmology (Princeton University Press, Princeton, NJ, 1993).

[20] B. Ryden, Introduction to Cosmology, 2nd ed. (Cambridge University Press, Cambridge, England, 2016).

[21] L. Jiang et al., Evidence for GN-Z11 as a luminous galaxy at redshift 10.957, Nat. Astron. 5, 256 (2021).

[22] P. A. Oesch et al., A remarkably luminous galaxy at $Z=11.1$ measured with Hubble Space Telescope Grism spectroscopy, Astrophys. J. 819, 129 (2016).

[23] P. A. R. Ade et al. (Planck Collaboration), Planck 2015 results. XIII. Cosmological parameters, Astron. Astrophys. 594, A13 (2016).

[24] G. R. Blumenthal, S. M. Faber, J. R. Primack, and M. J. Rees, Formation of galaxies and large-scale structure with cold dark matter, Nature (London) 311, 517 (1984).

[25] P. Bull et al., Beyond $\Lambda$ CDM: Problems, solutions, and the road ahead, Phys. Dark Universe 12, 56 (2016).

[26] A. Einstein, Die Feldgleichungen Der Gravitation, Sitzungsberichte Der Königlich Preußischen Akademie Der Wissenschaften (Berlin), Seite 844 (1915). 
[27] A. Friedmann, Über Die Krümmung Des Raumes, Z. Physik 10, 377 (1922).

[28] A. Friedmann, Über die Möglichkeit einer Welt mit konstanter negativer Krümmung des Raumes, Z. Physik 21, 326 (1924).

[29] E. Hubble, A Relation between distance and radial velocity among extragalactic nebulae, Proc. Natl. Acad. Sci. U.S.A. 15, 168 (1929).

[30] G. Lemaître, Expansion of the Universe, a homogeneous universe of constant mass and increasing radius accounting for the radial velocity of extra-galactic nebulae, Mon. Not. R. Astron. Soc. 91, 483 (1931).

[31] M. Davis, J. Huchra, D. W. Latham, and J. Tonry, A survey of galaxy redshifts. II-the large scale space distribution, Astrophys. J. 253, 423 (1982).

[32] J. Huchra, M. Davis, D. Latham, and J. Tonry, A survey of galaxy redshifts. IV-the data, Astrophys. J. Suppl. Ser. 52, 89 (1983).

[33] M. Colless et al., The 6dF galaxy survey: Final redshift release (DR3) and southern large-scale structures, Mon. Not. R. Astron. Soc. 399, 683 (2009).

[34] M. R. Blanton et al., Sloan digital sky survey IV: Mapping the Milky Way, nearby galaxies, and the distant universe, Astrophys. J. 154, 28 (2017).

[35] M. Skrutskie et al., The two micron all sky survey (2MASS), Astron. J. 131, 1163 (2006).

[36] A. A. Penzias and R.W. Wilson, A measurement of excess antenna temperature at $4080 \mathrm{Mc} / \mathrm{s}$., Astrophys. J. 142, 419 (1965).

[37] D. N. Spergel et al., First-Year Wilkinson microwave anisotropy probe (WMAP) observations: Determination of cosmological parameters, Astrophys. J. Suppl. Ser. 148, 175 (2003).

[38] M. A. K. Halliday, Language as Social Semiotic: The Social Interpretation of Language and Meaning (Edward Arnold, London, 1978).

[39] R. Hodge and G. Kress, Social Semiotics (Cornell University Press, Ithaca, NY, 1988).

[40] G. R. Kress and T. van Leeuwen, Reading Images: The Grammar of Visual Design (Psychology Press, London, 1996).

[41] G. R. Kress, Multimodality: A Social Semiotic Approach to Contemporary Communication (Routledge, London, New York, 2010).

[42] J. Airey and C. Linder, Social semiotics in university physics education, in Multiple Representations in Physics Education (Springer, Cham, 2017), pp. 95-122.

[43] J. L. Lemke, Social semiotics and science education, Am. J. Semiotics 5, 217 (1987), http://search.proquest .com/docview/213748355/abstract/41EA2A5CDB0C4C $\mathrm{C} 4 \mathrm{PQ} / 3$.

[44] T. van Leeuwen, Introducing Social Semiotics (Routledge, London, 2005).

[45] U. Eriksson, Reading the sky: From starspots to spotting stars, Doctoral thesis, Uppsala University, 2014, http://urn .kb.se/resolve?urn=urn:nbn:se:hkr:diva-13268.

[46] U. Eriksson, C. Linder, J. Airey, and A. Redfors, Introducing the anatomy of disciplinary discernment: An example from astronomy, Eur. J. Sci. Math. Educ. 2, 167 (2014).
[47] A. A. diSessa and B. L. Sherin, Meta-representation: An introduction, J. Math. Behav. 19, 385 (2000).

[48] R. Kozma and J. Russell, Students becoming chemists: Developing representational competence, in Visualization in Science Education, edited by J. K. Gilbert (Springer Netherlands, Dordrecht, 2005), pp. 121-145.

[49] P.-O. Wickman, Aesthetic Experience in Science Education: Learning and Meaning-Making As Situated Talk and Action (Routledge, Mahwah, NJ, 2006).

[50] D. Gentner, Structure-mapping: A theoretical framework for analogy, Cogn. Sci. 7, 155 (1983).

[51] J. Airey and C. Linder, A disciplinary discourse perspective on university science learning: Achieving fluency in a critical constellation of modes, J. Res. Sci. Teach. 46, 27 (2009)

[52] V. Prain and R. Tytler, Learning through constructing representations in science: A framework of representational construction affordances, Int. J. Sci. Educ. 34, 2751 (2012).

[53] M. J. Ford and E. A. Forman, Chapter 1: Redefining disciplinary learning in classroom contexts, Rev. Res. Educ. 30, 1 (2006).

[54] R. Lehrer and L. Schauble, Seeding evolutionary thinking by engaging children in modeling its foundations, Sci. Educ. 96, 701 (2012).

[55] P. Hubber and R. Tytler, Enacting a representation construction approach to teaching and learning astronomy, in Multiple Representations in Physics Education (Springer, Cham, 2017), pp. 139-161.

[56] P. Hubber and R. Tytler, Models and Learning Science, in Constructing Representations to Learn in Science (SensePublishers, Rotterdam, 2013), pp. 109-133.

[57] S. W. Gilbert, Model building and a definition of science, J. Res. Sci. Teach. 28, 73 (1991).

[58] A. G. Harrison and D. F. Treagust, Teaching and learning with analogies, in Metaphor and Analogy in Science Education, edited by P. J. Aubusson, A. G. Harrison, and S. M. Ritchie (Springer Netherlands, Dordrecht, 2006), pp. 11-24.

[59] S. W. Gilbert, Models-Based Science Teaching (NSTA Press, Arlington, VA, 2011).

[60] D. Gooding, Visualisation, inference and explanation in the sciences, in Studies in Multidisciplinarity, edited by G. Malcolm (Elsevier, 2005), Vol. 2, pp. 1-25.

[61] J. K. Gilbert, Visualization: A metacognitive skill in science and science education, in Visualization in Science Education (Springer, Dordrecht, 2005), pp. 9-27.

[62] Science Teachers' Use of Visual Representations, edited by B. Eilam and J. K. Gilbert (Springer International Publishing, New York, 2014).

[63] T. Fredlund, J. Airey, and C. Linder, Exploring the role of physics representations: An illustrative example from students sharing knowledge about refraction, Eur. J. Phys. 33, 657 (2012).

[64] U. Eriksson, Disciplinary discernment: Reading the sky in astronomy education, Phys. Rev. Phys. Educ. Res. 15, 010133 (2019).

[65] J. Airey, Social Semiotics in Higher Education: Examples from Teaching and Learning in Undergraduate Physics (Swedish Foundation for International 
Cooperation in Research in Higher Education (STINT), Sweden, 2015), p. 103, http://urn.kb.se/resolve?urn=urn: nbn:se:uu:diva-266049.

[66] M. Lynch and S. Y. Edgerton, Aesthetics and digital image processing: Representational craft in contemporary astronomy, Sociological Rev. 35, 184 (1987).

[67] T. A. Rector, Z. G. Levay, L. M. Frattare, K. K. Arcand, and M. Watzke, The aesthetics of astrophysics: How to make appealing color-composite images that convey the science, Publ. Astron. Soc. Pac. 129, 058007 (2017).

[68] S. Salimpour, Capturing the cosmos: Teaching astronomy (and more) through astrophotography in middle school, Robotic Telescopes, Student Research, and Education Proceedings 2, 1 (2019).

[69] S. Salimpour, Capturing the Cosmos: Art, Science or Both?, MSc Project Report, Swinburne University of Technology, 2014.

[70] J. English, Canvas and cosmos: Visual art techniques applied to astronomy data, Int. J. Mod. Phys. D 26, 1730010 (2017).

[71] J. Dewey, Art as Experience (Paragon Books, New York, 1979).

[72] J. Dewey, Aesthetic experience as a primary phase and as an artistic development, J. Aesthetics Art Criticism 9, 56 (1950).

[73] J. Dewey, The aesthetic element in education, in The Early Works of John Dewey, Volume 5, 1882-1898: Early Essays, 1895-1898 (Republished 1972), edited by J. A. Boydston, 1st ed. (Southern Illinois University Press, Carbondale, IL, 1897).

[74] J. Lemke, Feeling and meaning: A unitary bio-semiotic account, in International Handbook of Semiotics, edited by P. P. Trifonas (Springer Netherlands, Dordrecht, 2015), pp. 589-616.

[75] D. Malin and E. E. A. Slarke, Night Skies: The Art of Deep Space: An Exhibition of Astronomical Photographs, edited by N. S. W. Edgecliff and N. S. W. Epping (British Council, Anglo-Australian Observatory 1996).

[76] D. F. Treagust, A. G. Harrison, and G. J. Venville, Teaching science effectively with analogies: An approach for preservice and inservice teacher education, J. Sci. Teach. Educ. 9, 85 (1998).

[77] A. G. Harrison and D. F. Treagust, Secondary students' mental models of atoms and molecules: Implications for teaching chemistry, Sci. Educ. 80, 509 (1996).

[78] R. Duit, On the role of analogies and metaphors in learning science, Sci. Educ. 75, 649 (1991).

[79] W. F. McComas, Analogies in science teaching, in The Language of Science Education: An Expanded Glossary of Key Terms and Concepts in Science Teaching and Learning, edited by W. F. McComas (SensePublishers, Rotterdam, 2014), pp. 6-6.

[80] S. M. Ritchie, A. Bellocchi, H. Poltl, and M. Wearmouth, Metaphors and analogies in transition, in Metaphor and Analogy in Science Education, edited by P. J. Aubusson, A. G. Harrison, and S. M. Ritchie (Springer Netherlands, Dordrecht, 2006), pp. 143-153.

[81] D. Heywood, The place of analogies in science education, Cambridge J. Educ. 32, 233 (2002).
[82] J. Wiley, A. J. Jaeger, A. R. Taylor, and T. D. Griffin, When analogies harm: The effects of analogies on metacomprehension, Learning Instr. 55, 113 (2017).

[83] B. G. Glaser and A. L. Strauss, The Discovery of Grounded Theory: Strategies for Qualitative Research (Aldine, London, 1967).

[84] J. Corbin and A. Strauss, Basics of Qualitative Research: Techniques and Procedures for Developing Grounded Theory, 4th ed. (SAGE Publications, Inc, Los Angeles, 2014).

[85] Multimodal Teaching and Learning: The Rhetorics of the Science Classroom, edited by C. Jewitt, G. R. Kress, J. Ogborn, and C. Tsatsarelis (Bloomsbury Academic, 2014).

[86] C. Jewitt, J. J. Bezemer, and K. L. O'Halloran, Introducing Multimodality (Routledge, London, New York, 2016).

[87] W. J. Gibson and A. Brown, Working with Qualitative Data (SAGE, Thousand Oaks, CA, 2009).

[88] G. Kress and T. van Leeuwen, Structures of visual representation, J. Literary Semantics 21, 91 (2009).

[89] C. S. Peirce, The Essential Peirce, Volume 1: Selected Philosophical Writings. 1867-1893 (Indiana University Press, Bloomington, 1992).

[90] C. S. Peirce, The Essential Peirce, Volume 2: Selected Philosophical Writings, 1893-1913 (Indiana University Press, Bloomington, 1998).

[91] S. Salimpour, R. Tytler, and M. T. Fitzgerald, Exploring the cosmos: The challenge of identifying patterns and conceptual progressions from student survey responses in cosmology, in Methodological Approaches to STEM Education Research, edited by R. Tytler, P. White, J. Ferguson, and J. C. Clark (Cambridge Scholars Publishing, Cambridge, England, 2020), Vol. 1, https://www .cambridgescholars.com/product/978-1-5275-5551-8.

[92] V. Braun and V. Clarke, Using thematic analysis in psychology, Qualitative Res. Psychol. 3, 77 (2006).

[93] J. Saldaña, The Coding Manual for Qualitative Researchers, 3rd ed. (SAGE, London, 2016).

[94] W. Xu and K. Zammit, Applying Thematic Analysis to Education: A Hybrid Approach to Interpreting Data in Practitioner Research, Int. J. Qualitative Methods 19 (2020)

[95] C. O'Connor and H. Joffe, Intercoder Reliability in Qualitative Research: Debates and Practical Guidelines, Int. J. Qualitative Methods 19 (2020).

[96] K. Svensson and U. Eriksson, Concept of a Transductive Link, Phys. Rev. Phys. Educ. Res. 16, 026101 (2020).

[97] C. Linder, Disciplinary discourse, representation, and appresentation in the teaching and learning of science, Eur. J. Sci. Math. Educ. 1, 7 (2013).

[98] D. Gentner, Analogical Inference and Analogical Access (Illinois University at Urbana, Department of Computer Science, Urbana, IL, 1987).

[99] M. B. Hesse and M. B. Hesse, Models and Analogies in Science (University of Notre Dame Press, South Bend, IN, 1966)

[100] G. J. Venville and D. F. Treagust, The role of analogies in promoting conceptual change in Biology, Instr. Sci. 24, 295 (1996).

[101] R. Freedman, R. Geller, and W. J. Kaufmann, Universe, 9th ed. (W. H. Freeman and Company, San Francisco, CA, 2011). 
[102] A. Fraknoi, D. Morrison, and S. Wolff, OpenStax Astronomy Textbook (OpenStax, 2020), https://openstax .org/details/books/astronomy.

[103] A. G. Lemaître and A.S. Eddington, The expanding Universe, Mon. Not. R. Astron. Soc. 91, 490 (1931).

[104] R. A. Knop et al., New constraints on $\Omega \mathrm{m}, \Omega \lambda$, and $\mathrm{w}$ from an independent set of 11 high-redshift supernovae observed with The Hubble Space Telescope, Astrophys. J. 598, 102 (2003).

[105] J. R. Pritchard and A. Loeb, Constraining the unexplored period between the dark ages and reionization with observations of the global $21 \mathrm{~cm}$ signal, Phys. Rev. D 82, 023006 (2010).

[106] J. Pritchard and A. Loeb, Hydrogen was not ionized abruptly, Nature (London) 468, 772 (2010).

[107] W. Hu and M. White, The cosmic symphony, in Scientific American (2004), https://doi.org/10.1038/scientificamerican0204-44.

[108] J. Airey and U. Eriksson, Unpacking the HertzsprungRussell diagram: A social semiotic analysis of the disci- plinary and pedagogical affordances of a central resource in astronomy, Designs for Learning 11, 99 (2019).

[109] M. E. Lira and M. Stieff, Using gesture analysis to assess students' developing representational competence, in Towards a Framework for Representational Competence in Science Education, edited by K. L. Daniel (Springer International Publishing, Cham, 2018), pp. 205-228.

[110] A. A. diSessa, Metarepresentation: Native competence and targets for instruction, Cognit. Instr. 22, 293 (2004).

[111] R. Tytler and V. Prain, Representation construction to support conceptual change, in International Handbook of Research on Conceptual Change, edited by S. Vosniadou, 2nd ed. (Routledge, New York, 2013), pp. 1009-1042, https://doi.org/10.4324/9780203154472.

[112] J. Ojala, The Third Planet, Int. J. Sci. Educ. 14, 191 (1992).

[113] I. Testa, S. Leccia, and E. Puddu, Astronomy textbook images: Do they really help students?, Phys. Educ. 49, 332 (2014). 